\title{
Article
}

\section{Non-Covalent Interactions and Impact of Charge Penetration Effects in Linear Oligoacene Dimers and Single Crystals}

Sean M Ryno, Chad Risko, and Jean-Luc Bredas

Chem. Mater., Just Accepted Manuscript • DOI: 10.1021/acs.chemmater.6b01340 • Publication Date (Web): 18 May 2016

Downloaded from http://pubs.acs.org on May 24, 2016

\section{Just Accepted}

"Just Accepted" manuscripts have been peer-reviewed and accepted for publication. They are posted online prior to technical editing, formatting for publication and author proofing. The American Chemical Society provides "Just Accepted" as a free service to the research community to expedite the dissemination of scientific material as soon as possible after acceptance. "Just Accepted" manuscripts appear in full in PDF format accompanied by an HTML abstract. "Just Accepted" manuscripts have been fully peer reviewed, but should not be considered the official version of record. They are accessible to all readers and citable by the Digital Object Identifier (DOI®). "Just Accepted" is an optional service offered to authors. Therefore, the "Just Accepted" Web site may not include all articles that will be published in the journal. After a manuscript is technically edited and formatted, it will be removed from the "Just Accepted" Web site and published as an ASAP article. Note that technical editing may introduce minor changes to the manuscript text and/or graphics which could affect content, and all legal disclaimers and ethical guidelines that apply to the journal pertain. ACS cannot be held responsible for errors or consequences arising from the use of information contained in these "Just Accepted" manuscripts. 


\title{
Non-Covalent Interactions and Impact of Charge Penetration
} Effects in Linear Oligoacene Dimers and Single Crystals

\author{
Sean M. Ryno," Chad Risko, ${ }^{\#, *}$ and Jean-Luc Brédas,* \\ "Solar and Photovoltaics Engineering Research Center \\ King Abdullah University of Science and Technology \\ Thuwal 23599-6900, Kingdom of Saudi Arabia \\ \#Department of Chemistry \& \\ Center for Applied Energy Research \\ University of Kentucky \\ Lexington, Kentucky 40506-0055
}

*Corresponding authors: chad.risko@kyu.edu ; jean-luc.bredas@kaust.edu.sa 


\begin{abstract}
Non-covalent interactions determine in large part the thermodynamic aspects of molecular packing in organic crystals. Using a combination of symmetry-adapted perturbation theory (SAPT) and classical multipole electrostatics, we describe the interaction potential energy surfaces for dimers of the oligoacene family, from benzene to hexacene, including up to 5000 configurations for each system. An analysis of these surfaces and a thorough assessment of dimers extracted from the reported crystal structures underline that high-order interactions (i.e., three-body non-additive interactions) must be considered in order to rationalize the details of the crystal structures. A comparison of the SAPT electrostatic energy with the multipole interaction energy demonstrates the importance of the contribution of charge penetration, which is shown to account for up to $50 \%$ of the total interaction energy in dimers extracted from the experimental single crystals; in the case of the most stable co-facial model dimers, this contribution is even larger than the total interaction energy. Our results highlight the importance of taking account of charge penetration in studies of the larger oligoacenes.
\end{abstract}




\section{Introduction}

In organic molecular crystals, like those derived from $\pi$-conjugated molecules such as sexithienyl or the oligoacenes (benzene, naphthalene, anthracene, tetracene, pentacene, and hexacene), the molecules generally interact through weak van der Waals forces. ${ }^{1}$ Chemical substitution/functionalization, either within the conjugated backbone or along the periphery, can greatly impact these non-covalent interactions (for instance, through increased/decreased $\pi$ conjugation, introduction of dipole moments, or modulation in the sign or magnitude of multipole moments) and result in substantial changes in solid-state molecular packing and materials properties. ${ }^{2-3}$ Given their importance, a fundamental understanding of the non-covalent interactions among $\pi$-conjugated molecules would be a key step towards the more comprehensive understanding required to synthetically design novel molecular-based materials systems. Here, by applying symmetry-adapted perturbation theory (SAPT) to a series of $\pi$ conjugated molecules, we develop insight into the evolution of non-covalent interactions upon various geometric transformations.

Sherrill, Wheeler, and others have shown that a simple electrostatic picture is not enough to accurately describe the molecular interactions that drive the solid-state packing of $\pi$-conjugated systems, as dispersion effects dominate the interactions. ${ }^{4-8}$ Recently, additional effects due to charge penetration - the electron-nucleus attraction due to the reduced screening of the nuclei of one monomer by its electrons as the electron clouds of two monomers overlap - have been shown to be significant at typical intermolecular separations in organic crystals. ${ }^{9-15}$ In fact, without the inclusion of explicit charge penetration, ${ }^{16}$ force fields using atom-centered charge models and semi-empirical models where only electrostatic interactions are considered for the 
environment of a given subsystem, are of limited use to describe the complex interactions in noncovalently bound systems, especially for non-polar materials. ${ }^{9}$ We note that a number of force fields that include explicit terms for charge penetration have been reported or are currently in development. $^{4,17-21}$

In attempts to understand the packing behavior of oligoacenes in the solid state, a number of investigations have explored the nature and strength of the non-covalent interactions. ${ }^{6,8,22-34}$ These investigations generally have focused on a few representative dimer configurations (see Figure 1), in particular the cofacial (eclipsed or parallel-displaced) and T-shaped configurations, or only on the separation distance between dimers, though a few studies have also explored broader potential energy surfaces for oligoacene interactions as a function of the oligoacene length. ${ }^{33-34}$ This issue is of interest since the crystalline packings change with oligoacene length, which is indicative of modifications in the non-covalent interactions.
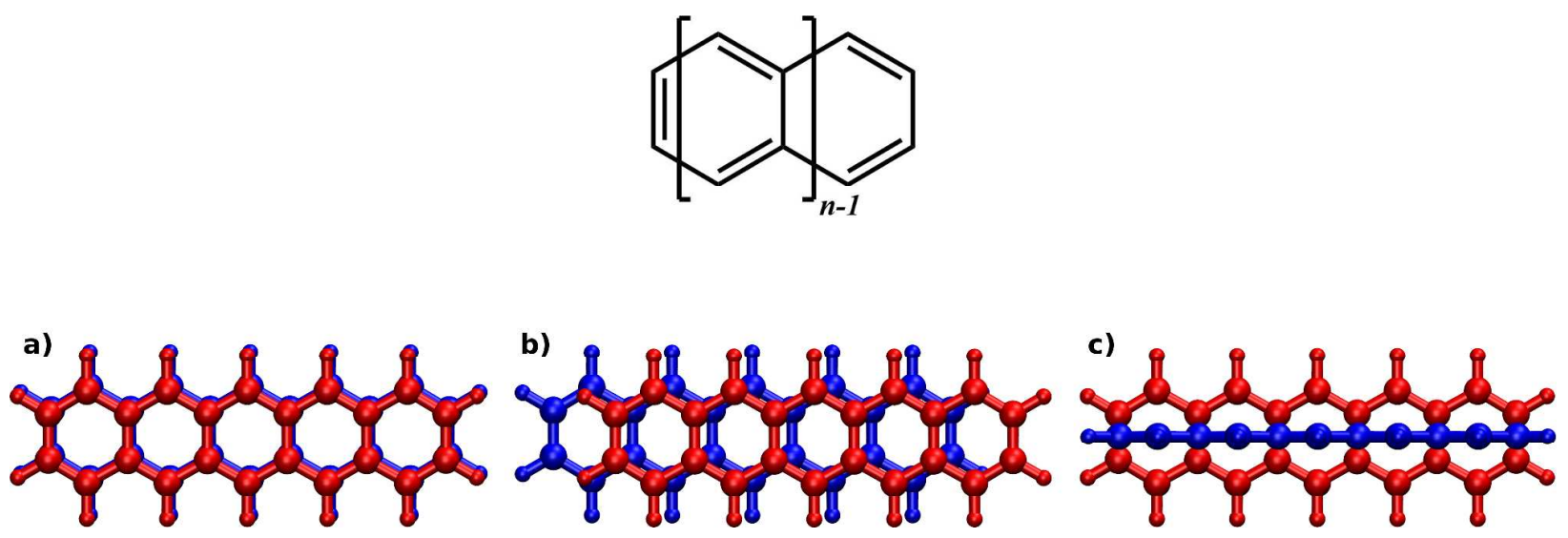
Figure 1. (Top) Chemical structure of linear oligoacenes, where $n$ is the number of fused rings, i.e., $n=1$ for benzene and $n=5$ for pentacene. Illustration of the pentacene dimer with: (a) an eclipsed configuration; (b) a parallel-displaced configuration; and (c) a T-shaped configuration.

By applying SAPT to dimers of benzene, naphthalene, anthracene, tetracene, pentacene, and hexacene, we explore the influence of acene length on the non-covalent interactions of the oligoacenes and make connections with preferred crystal packing configurations. For each of the systems, we consider three dimer transformations related to: (i) angle between monomers, where we vary the dimers from a co-facial arrangement to a T-shaped configuration; (ii) intermolecular separation, where we vary the separation distance from $2.8 \AA$ to $5.0 \AA ;^{35}$ and (iii) translation, where a molecule in the dimer is moved along its long-axis from an eclipsed configuration to one of no molecular overlap (see Figure 2). From these transformations, we construct potential energy surfaces of the individual transformations and select combinations of transformations to extract trends in the non-covalent interactions. Additionally, we examine the charge penetration contribution to the interaction energy as a function of acene length. We observe that the charge penetration increases linearly with acene length and becomes significant at increasing distances for longer acenes. 

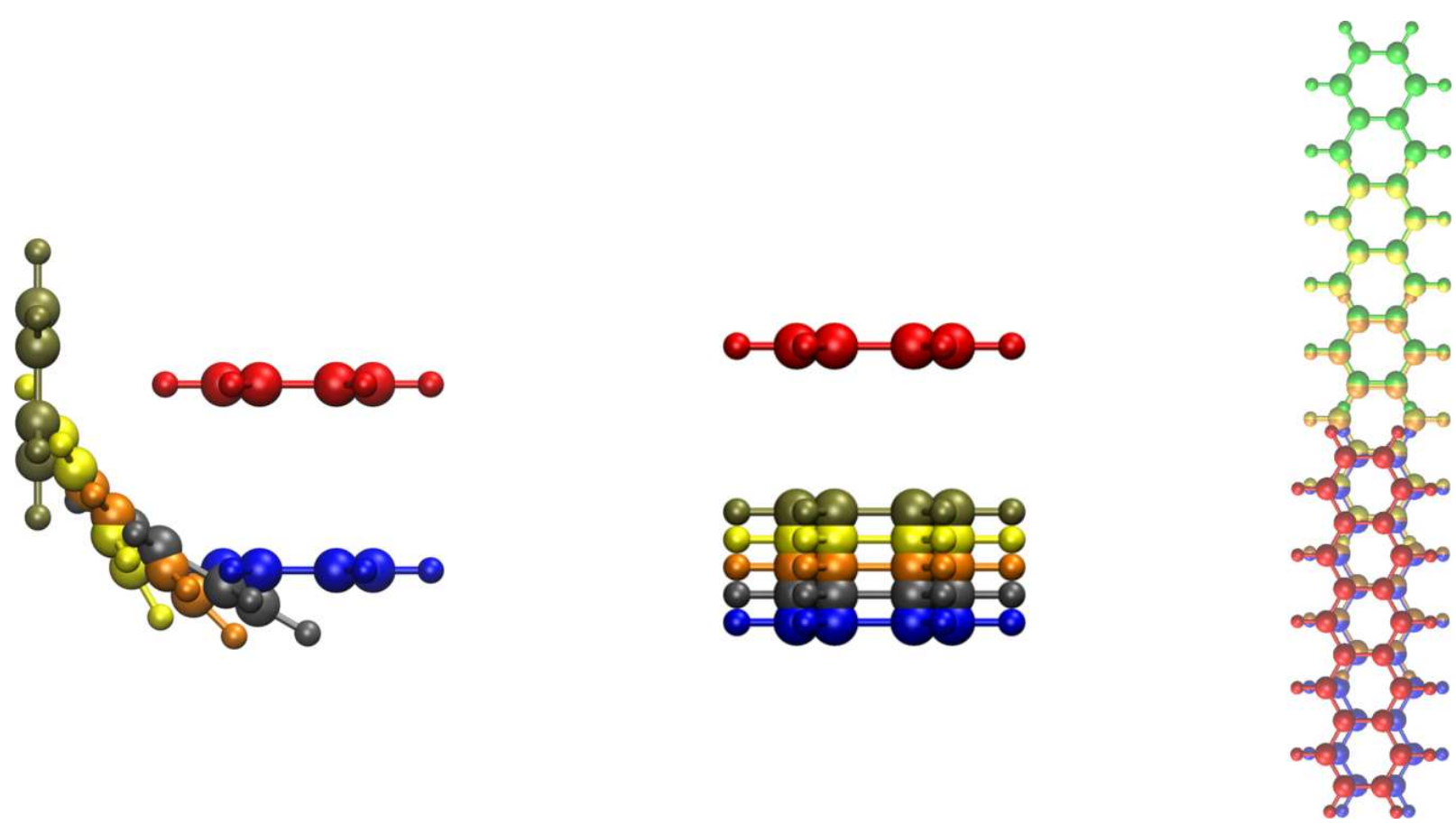

Figure 2. (Left) Ball-and-stick representation of pentacene as a function of angle between monomers. Ball-and-stick representations of pentacene displaying increasing intermolecular separation (center) and long-axis translation (right) as the second monomer moves from an eclipsed configuration to no-overlap. Note that in all instances only one of the monomers is shown moving (and adopts colors from blue to green) while the other monomer (in red) remains at a fixed position.

\section{Computational Methodology}

At the electronic-structure level, the evaluation of non-covalent systems is often limited to methods that utilize empirical dispersion terms added to the final energy, such as in the density functional theory (DFT) plus dispersion $(\mathrm{DFT}+\mathrm{D})$ formulation, ${ }^{36-43}$ or to computationally expensive methods such as $\operatorname{CCSD}(\mathrm{T}) .{ }^{44}$ On the other hand, empirical dispersion corrections, such as Grimme's D2 or D3 corrections, ${ }^{37,45}$ including terms to model dispersion as $C_{6} / R^{6}$ and $C_{8} / R^{8}$, where $C_{n}$ is the dispersion coefficient and $R^{-n}$ is the distance dependence of a specific 
term between two atom centers, have been shown to underestimate the dispersion contribution by some $40 \%$ compared to wave function-based methods; ${ }^{11}$ on the other hand, highly accurate methods such as $\operatorname{CCSD}(\mathrm{T})$ are limited to system sizes of at most a few tens of atoms due to unfavorable scaling, $O\left(n^{7}\right)$. Because of these limitations, intermediate methods are often used, such as spin-component scaled MP2 (SCS-MP2); ${ }^{46-47}$ SCS-MP2 interaction energies have shown good agreement with $\operatorname{CCSD}(\mathrm{T})$ interaction energies (within less than $0.2 \mathrm{kcal} / \mathrm{mol}$ error), ${ }^{48}$ we note that SCS-MP2 is parameterized using $\operatorname{CCSD}(\mathrm{T})$ reaction energies fit to a test set.

Each of the above methods uses a supermolecular approach, where the interaction energy is the difference between the energy of a dimer or complex consisting of two molecules and the energies of the isolated molecules. This method, however, relies on the subtraction of two very large values to obtain a relatively small value, where the error associated with the large values can be greater than the desired quantity. To avoid this issue, the interaction energy can also be treated as a perturbation to the Hamiltonian of the individual monomers. The most widespread perturbative approach is symmetry-adapted perturbation theory (SAPT), ${ }^{32}$ which has the additional advantage compared to supermolecular approaches of allowing for a decomposition of the interaction energy into physically meaningful components: exchange, dispersion, permanent electrostatics, and induction; more thorough discussions of these components are available in the literature $^{11,32,49}$ (note that there exist other energy decomposition analysis schemes, such as that derived by Morokuma ${ }^{50-51}$ and applied to SCS-MP2 interaction energies by Grimme). ${ }^{52}$

All SAPT calculations were completed at the SAPT0/jun-cc-pvdz level (hereafter simply denoted as SAPT0) as implemented in the PSI4 program. ${ }^{53}$ This level of calculation has been dubbed the bronze-standard by Parker and co-workers ${ }^{54}$ as it allows for accurate calculations of 
interaction energies (mean absolute error of $0.49 \mathrm{kcal} / \mathrm{mol}$ for the $\mathrm{S} 22$ test set) in reasonable computational times; this requirement is necessary given the large number of configurations considered in the present work, up to 5000 points in the case of hexacene. Additional comparison of SAPT0 to SAPT(DFT) for co-facial and T-shaped benzene dimers (S3 and S8 from Ref. 55) results in errors of $0.12 \mathrm{kcal} / \mathrm{mol}$ and $0.47 \mathrm{kcal} / \mathrm{mol}$, respectively. The interaction energy is divided into four contributions: (i) exchange; (ii) electrostatics; (iii) induction, which contains the induction, exchange-induction, and delta-HF correction terms; and (iv) dispersion, which contains dispersion and exchange-dispersion. All distributed multipole analysis (DMA) calculations were carried out at the HF/6-311G(d,p) level as implemented in the Molpro software suite. ${ }^{56}$ Distributed multipoles were centered at each atom. The DMA data were then used to calculate multipole-multipole interaction energies through the 32-pole and up to rank 6 (i.e., $R^{-6}$ ) using a custom script based on the equations of Stone. ${ }^{57}$ The geometries of the crystal dimers were taken from the experimentally determined structures as reported in the Cambridge Structural Database without further modification: benzene (BENZEN15), ${ }^{58}$ naphthalene (NAPHTA06), ${ }^{59}$ anthracene (ANTCEN09), ${ }^{60}$ tetracene (TETCEN01), ${ }^{61}$ and pentacene (PENCEN04). ${ }^{62}$ The dimer geometry of crystalline hexacene was extracted from the recently reported crystal structure in Ref. 63. For tetracene, pentacene, and hexacene the dimers were constructed using both molecules in the unit cell.

The geometries of isolated benzene, naphthalene, anthracene, tetracene, pentacene, and hexacene molecules were optimized at the MP2/6-31+G(d,p) level as implemented in the Gaussian 09 Rev. B.01 software suite. ${ }^{64}$ For pentacene and hexacene, the enforcement of $\mathrm{D}_{2 \mathrm{~h}}$ symmetry was required to obtain minimized geometries. 
Dimers for the SAPT0 analyses were then created by duplicating the optimized single molecules (denoted as Molecule \#1) and transforming the position of Molecule \#2 through a broad spatial profile using a custom script. The following molecular transformations are considered:

- The intermolecular separation was varied in $0.1 \AA$ increments from $2.8 \AA$ to $5.0 \AA$, see Figure 2, center.

- Molecule \#2 was translated along the oligoacene long-axis, going from a perfectly eclipsed configuration to no spatial molecular overlap with Molecule \#1, in either $0.1 \AA$ or $1.0 \AA$ increments (from $0.0 \AA$ to $17.0 \AA$ for hexacene), see Figure 2, right.

- The intermonomer angle was varied from $0^{\circ}$ (co-facial) to $90^{\circ}$ (T-shaped) in $10^{\circ}$ increments, with the additional consideration of a $45^{\circ}$ dimer, see Figure 2, left. ${ }^{65}$

Note that all possible combinations of the above dimer configurations are considered, as this strategy allows for the creation of a multidimensional potential energy surface that can then be separated according to individual transformations or combinations of transformations. For each dimer configuration considered, both the SAPT0 interaction energy and multipole electrostatic interaction energy were determined.

As the DMA interaction between molecules corresponds to a description of the electrostatic term in which only the charge penetration is neglected, the charge penetration contribution to the interaction energy is calculated as the difference between the SAPT0 electrostatic interaction energy and the DMA electrostatic interaction energy (we note that some error is associated with this procedure due to the DMA truncation at the 32-pole and the differences in basis sets). The charge penetration contribution has been calculated only along the intermolecular separation coordinate, as this is where its impact should be most pronounced. 


\section{Results and Discussion}

\section{a) Interaction Energies of Crystal Geometry Dimers}

To provide a baseline for comparison, we first examine the non-covalent interactions in dimers extracted from the experimentally determined crystal structures (Table 1); for asymmetric unit cells, i.e., tetracene, pentacene, and hexacene, the dimer is composed of both molecules of the unit cell. As expected, we observe that the interaction energy increases as the acene length increases, e.g., by $\sim 220 \%$ from benzene to naphthalene and by $\sim 20 \%$ from pentacene to hexacene, see Table 1. The largest contribution to the interaction energy is dispersion, whose contribution is about $50 \%$ larger and of opposite sign as the exchange contribution, and generally displays the largest relative increase as the number of fused rings is increased. While their contributions are smaller, the electrostatic and induction contributions also rapidly increase as acene length increases, with individual contributions being of the same order of magnitude and often larger than the difference between the exchange and dispersion contributions, highlighting the importance of these terms to the overall interaction energy.

As stated earlier, the experimentally determined crystalline packings change with increasing acene length. For benzene, the dimer configuration is more similar to that of an ideal T-shaped dimer where the edge of one of the molecules is oriented towards the face of the other, with a Tshape angle of about $83^{\circ}$. For the other acenes, the picture is not as simple since naphthalene and anthracene show a rotation in the plane of the backbone of one molecule, such that the long-axes of the two molecules are not parallel. In addition, the angle between the longer acenes is reduced further from the idealized $90^{\circ}$ for a T-shaped dimer to between $50^{\circ}$ and $53^{\circ}$. This range of 
configurations necessitates that a large space must be sampled to fully understand how noncovalent interactions determine the crystalline packing morphologies.

Table 1. SAPT0/jun-cc-pvdz energy decomposition analysis components of oligoacene dimers extracted from their experimentally determined crystal structures. $E_{\text {SAPT } 0}$ is the total SAPT0 interaction energy; $E_{\text {elect }}, E_{\text {exch }}, E_{\text {ind }}$, and $E_{\text {disp }}$ are the electrostatic, exchange, induction, and dispersion contributions, respectively. All units in $\mathrm{kcal} / \mathrm{mol}$.

\begin{tabular}{l|ccccc}
\hline (kcal/mol) & $E_{\text {SAPTO }}$ & $E_{\text {elect }}$ & $E_{\text {ind }}$ & $E_{\text {disp }}$ & $E_{\text {exch }}$ \\
\hline Benzene & -2.41 & -1.19 & -0.25 & -2.81 & 1.85 \\
Naphthalene & -5.35 & -2.53 & -0.67 & -7.21 & 5.06 \\
Anthracene & -8.50 & -3.69 & -1.02 & -11.36 & 7.58 \\
Tetracene & -12.08 & -4.06 & -1.24 & -15.57 & 8.78 \\
Pentacene & -16.95 & -6.43 & -2.03 & -21.89 & 14.06 \\
Hexacene & -20.43 & -6.91 & -2.18 & -26.59 & 15.25 \\
\hline
\end{tabular}

\section{b) Evolution of the Intermolecular Interactions upon Dimer Transformation}

We now turn to the dimer configurations that are the primary interest of this investigation. Previously, the benzene through tetracene cases have been considered by Grimme ${ }^{52}$ to determine if "special" $\pi-\pi$ interactions are present in stacked aromatic complexes that are not present in saturated hydrocarbons, while benzene through pentacene were considered by Sherrill ${ }^{11}$ in an application of density fitting to SAPT and to further investigate $\pi-\pi$ interactions. Both of these studies, though, focused on only a few possible dimer configurations, i.e., ideal T-shaped, 
eclipsed, or parallel-displaced (Figure 1). The work of Podeszwa and Szalewicz mapped comprehensive six-dimensional potential energy surfaces, but this study was restricted to benzene, naphthalene, and anthracene, making it difficult to determine packing effects as a function of acene length. ${ }^{33-34}$ As noted above, the packing in the solid state is much more complex than these idealized configurations and it is of interest to understand what drives the preferred packing orientations. In order to provide a more complete picture, we investigate here a very large number of configurations, extending into the thousands.

Before looking at trends upon specific transformations, it is important to determine the lowest energy structures for both co-facial and T-shaped arrangements along the potential energy surface for the sake of comparison to prior investigations, see Table 2. Note that the lowest energy dimer configurations are not equivalent for each system: e.g., co-facial benzene prefers a $3.5 \AA$ interplanar separation with a $1.7 \AA$ long-axis translation, while in pentacene the molecular planes are separated by $3.4 \AA$ with a $1.3 \AA$ translation; a comparison of dimers in equivalent arrangements will be made later. It is interesting to note that the preferred long-axis translations of one molecule of the dimer species do not appear to correspond to any specific geometric value, such as the midpoint of the fused rings $(1.21 \AA)$, with optimal displacements generally larger.

For each of the acene systems, we find that the co-facial arrangement is preferred over the Tshaped configuration and that the interaction energy difference between co-facial and T-shaped increases non-linearly with acene length. This agrees well with previous results for benzene, where the co-facial benzene dimer was estimated to be about $0.1 \mathrm{kcal} / \mathrm{mol}$ more stable than the $\mathrm{T}$-shaped dimer; ${ }^{33}$ the global minimum of benzene has been reported as a twisted T-shaped dimer. ${ }^{33,66}$ The primary contributions to the energy difference between the configurations are the 
dispersion component (larger for the co-facial dimers due to the increased degree of interaction of the $\pi$-clouds) and the electrostatic component, with both providing approximately equal contributions. The electrostatic contribution is perhaps the more interesting to consider since there is a qualitative difference between the multipole-only-based electrostatic interaction and the SAPT0-determined electrostatic interaction for the co-facial arrangement. This is due mainly to charge penetration, resulting from the overlap of the $\pi$-clouds at relatively small intermolecular separations. Additionally, note that as the number of fused rings increases, the DMA electrostatic energy increases by a relatively small amount $(0.5 \mathrm{kcal} / \mathrm{mol} / \mathrm{ring})$ compared to the SAPT0 electrostatic energy $(1.5-2.0 \mathrm{kcal} / \mathrm{mol} / \mathrm{ring})$, again due to the large contribution of charge penetration at short intermolecular distances, vide infra.

Table 2. SAPT0 energy components and DMA electrostatic interaction energies of the lowestenergy oligoacene dimers in co-facial $\left(0^{\circ}\right)$ and T-shaped $\left(90^{\circ}\right)$ arrangements. $E_{S A P T 0}$ is the total SAPT0 interaction energy; $E_{\text {elect }}, E_{\text {exch }}, E_{\text {ind }}$, and $E_{\text {disp }}$ are the electrostatic, exchange, induction, and dispersion contributions, respectively, and $E_{d m a}$ is the multipole-multipole electrostatic interaction energy. Values in parentheses present the separation distance and long-axis translation (in Angstroms), respectively. All energy data are in kcal/mol.

\begin{tabular}{l|cccccc}
\hline (kcal/mol) & $E_{\text {SAPTO }}$ & $E_{\text {elect }}$ & $E_{\text {ind }}$ & $E_{\text {disp }}$ & $E_{\text {exch }}$ & $E_{\text {dma }}$ \\
\hline & -2.82 & -1.645 & -0.73 & -6.72 & 6.28 & 1.34 \\
$\begin{array}{l}\text { Benzene } \\
(3.5 / 1.7)\end{array}$ & -7.58 & -3.45 & -1.23 & -15.00 & 12.10 & 2.22 \\
$\begin{array}{l}\text { Naphthalene } \\
(3.5 / 1.5)\end{array}$ & -13.47 & -7.78 & -2.32 & -27.34 & 23.96 & 3.93 \\
$\begin{array}{l}\text { Anthracene } \\
(3.4 / 1.4)\end{array}$ & -19.16 & -10.30 & -3.06 & -37.43 & 31.63 & 4.08 \\
$\begin{array}{l}\text { Tetracene } \\
(3.4 / 1.3)\end{array}$ & & & & & & \\
\hline
\end{tabular}




\begin{tabular}{|c|c|c|c|c|c|c|}
\hline $\begin{array}{l}\text { Pentacene } \\
(3.4 / 1.3)\end{array}$ & -25.28 & -12.79 & -3.81 & -47.70 & 39.03 & 4.88 \\
\hline $\begin{array}{l}\text { Hexacene } \\
(3.4 / 1.3)\end{array}$ & -31.65 & -15.34 & -4.60 & -58.22 & 46.51 & 5.69 \\
\hline & \multicolumn{6}{|c|}{ T-shaped } \\
\hline $\begin{array}{l}\text { Benzene } \\
(3.5 / 0)\end{array}$ & -2.82 & -1.80 & -0.53 & -3.79 & 3.29 & -0.67 \\
\hline $\begin{array}{l}\text { Naphthalene } \\
(3.5 / 0)\end{array}$ & -5.88 & -3.35 & -1.05 & -8.31 & 6.82 & -1.15 \\
\hline $\begin{array}{l}\text { Anthracene } \\
(3.5 / 0)\end{array}$ & -9.25 & -4.83 & -1.55 & -13.18 & 10.31 & -1.55 \\
\hline $\begin{array}{l}\text { Tetracene } \\
(3.5 / 0)\end{array}$ & -12.67 & -7.43 & -2.46 & -20.05 & 17.27 & -1.93 \\
\hline $\begin{array}{l}\text { Pentacene } \\
(3.5 / 0)\end{array}$ & -16.31 & -9.27 & -3.13 & -25.86 & 21.95 & -2.30 \\
\hline $\begin{array}{l}\text { Hexacene } \\
(3.5 / 0)\end{array}$ & -20.11 & -9.82 & -3.35 & -29.54 & 22.61 & -2.72 \\
\hline
\end{tabular}

We next evaluate how the potential-energy surfaces of different dimers change under specific transformations (Figures S1-S6). First, we considered the case where only the separation distance is changed. As expected, the interaction energy falls quickly as the separation distance increases beyond ca. $3.5 \AA{ }^{67}$ with the exchange term decaying fastest (exponential dependence), ${ }^{33}$ followed by electrostatics and induction interactions, ${ }^{68}$ and then dispersion interactions (approximately $R^{-6}$ dependence). Note that there is fast decay of the electrostatics at small separations due to the exponential dependence of the charge penetration on the density overlap. Dispersion dominates the interaction energy at separations greater than $4.5 \AA$ for co-facial arrangements and greater than $4.0 \AA$ for T-shaped dimers; at even larger separations (i.e., greater 
than $\sim 7 \AA$ ), the electrostatics again take over due to the $1 / R^{5}$ distance dependence of the quadrupole-quadrupole interactions compared to the approximately $1 / R^{6}$ distance dependence of dispersion. The slower fall-off of the dispersion for co-facial dimers may be attributed to the large interaction of the $\pi$-clouds, and while exchange and charge penetration also depend on $\pi$ cloud interactions, their contribution falls off exponentially as distance increases, i.e., much faster than dispersion, which evolves as a power series in the form $1 / R^{6}+1 / R^{8}+1 / R^{10}+\ldots .57$

If we start with dimers that are in an eclipsed, co-facial configuration (no long-axis translation), at $45^{\circ}$, and at $90^{\circ}$ (T-shaped) followed by a separation of the dimers, we observe a combination of the angle, separation, and length dependences, see Figures S7-S12. The $45^{\circ}$ dimer is the least stable configuration for each of the acenes, and has its lowest energy configuration at a larger separation $(3.8 \AA)$ than either the eclipsed or T-shaped orientations, thus limiting the stabilization due to charge penetration. Furthermore, as the acene length increases, the strongest interacting configuration changes. For example, for benzene, the T-shaped dimer is the most stable, with the curves for the $45^{\circ}$ and eclipsed dimers being approximately equal. As the number of fused rings is increased to anthracene, the eclipsed dimer curve becomes intermediate between those of the $45^{\circ}$ and $90^{\circ}$ dimers. Finally, for pentacene, the eclipsed curve is slightly more stable than the $90^{\circ}$ dimer, due to a combination of dispersion and electrostatics interactions.

From Figure 3, we observe that the potential-energy surfaces of benzene, anthracene, and pentacene, as the angle between the dimers is changed, are similar although the scales are different. For all systems, the evolution of $E_{S A P T 0}$ follows qualitatively those of exchange and electrostatics with minima at about $20^{\circ}$ and $90^{\circ}$ and a maximum at about $60^{\circ}$ (i.e., exchange is more repulsive when electrostatics are more attractive). Here, electrostatics are dominated by 
charge penetration at the intermolecular distances considered; thus, both exchange and electrostatics are dependent on the extent of orbital overlap with their contributions being of opposite sign.

It is noteworthy that the intermonomer angles found in the crystal structures $\left(\sim 50^{\circ}\right.$ to $\left.\sim 83^{\circ}\right)$ lie near the maximum of $E_{S A P T 0}$; these results suggest that these configurations should be less stable than other arrangements. That this is in fact not the case underlines that there exist important interactions in the bulk material that are not accounted for in two-body SAPT0, such as nonadditive three-body interactions. In benzene trimers, the three-body dispersion correction has been estimated to be in the range of $0.76 \mathrm{kcal} / \mathrm{mol}$ to $1.67 \mathrm{kcal} / \mathrm{mol}$, a significant contribution. ${ }^{69-}$ ${ }^{72}$ Indeed, Szalewicz has recently proposed a model to predict crystal packing that requires the inclusion of such non-additive terms. ${ }^{13}$

Interesting trends present themselves when we consider co-facial or T-shaped dimers at a fixed separation and displace one molecule of each dimer along the long axis (Figure 4). For each of the co-facial dimers, $E_{S A P T 0}, E_{\text {exch }}$, and $E_{\text {ind }}$ fluctuate with the number of maxima and minima corresponding to the number of fused rings (i.e., one maximum/minimum for benzene and five maxima/minima for pentacene), while $E_{\text {elect }}$ displays maxima and minima opposite the other contributions. The minima in total energy correspond to a staggered arrangement, where bridging carbon-carbon bonds are above the rings of the adjacent molecule. Similar trends are observed for the T-shaped dimers, although the energy difference between the maxima and minima are larger. This is due to the close contacts that occur when the peripheral hydrogens are pointed towards bridging carbon-carbon bonds. 

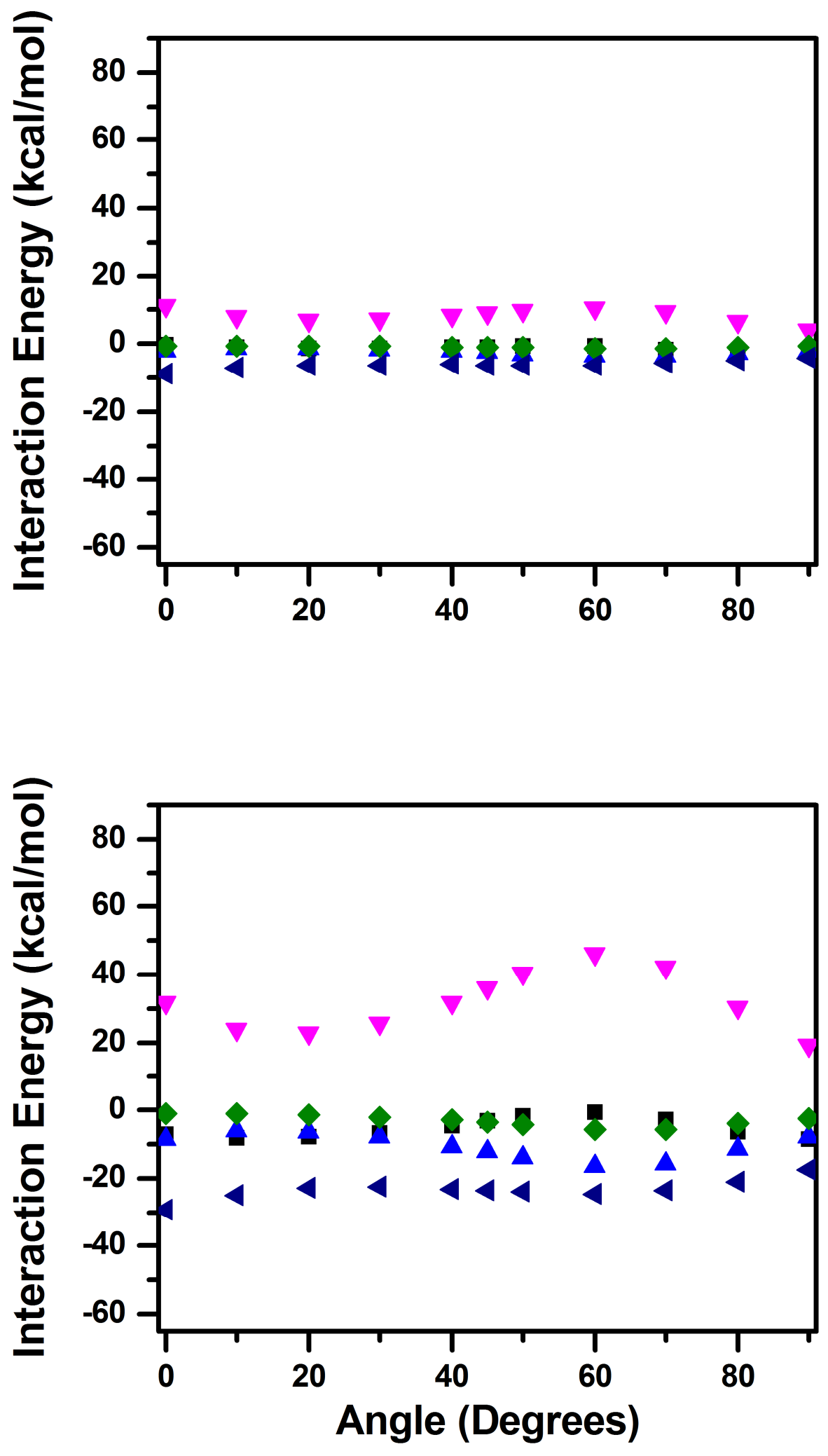

(Figure 3 continued on next page.) 


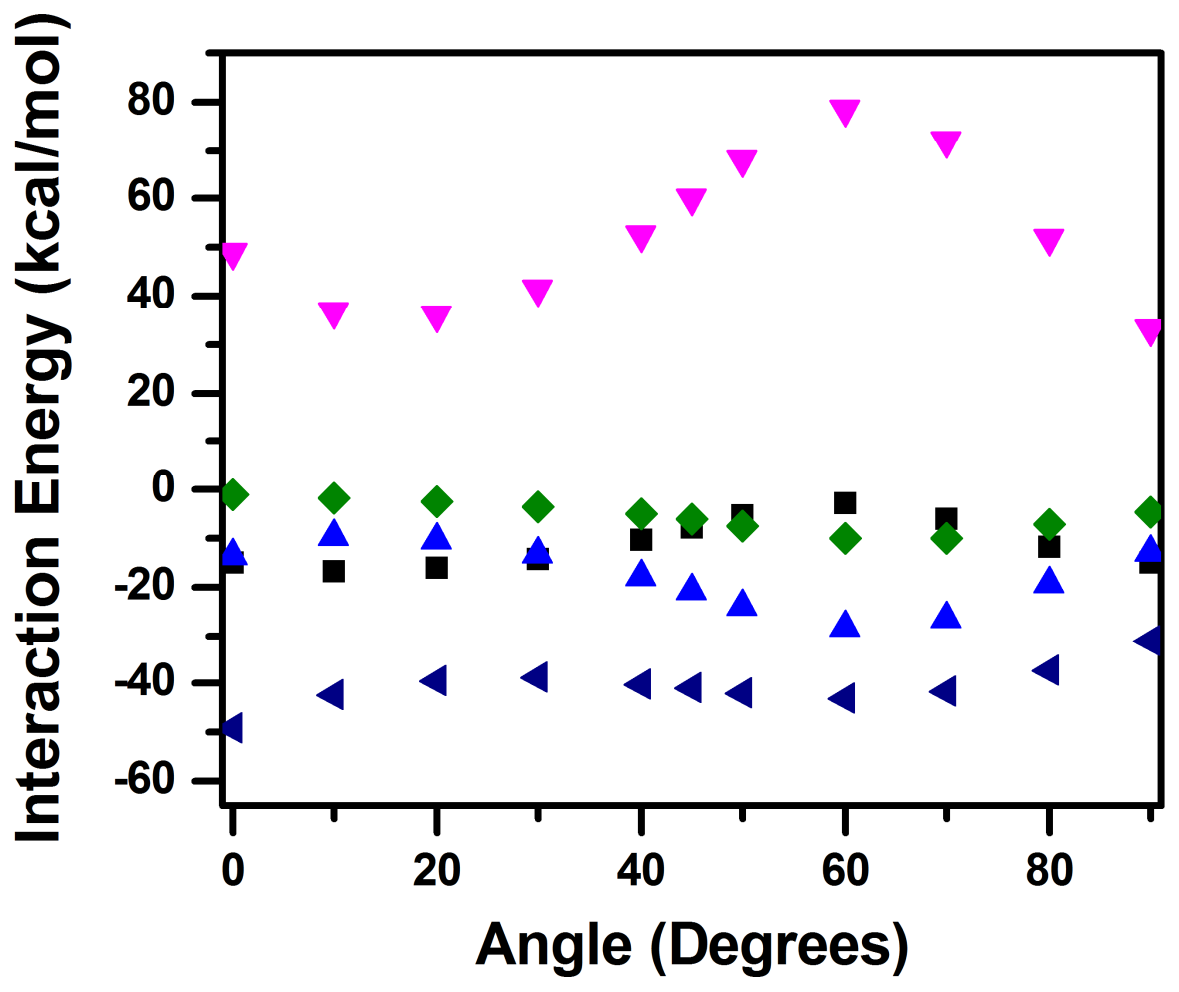

Figure 3. SAPT0 energy components for benzene (top), anthracene (center), and pentacene (bottom) at a separation distance of $3.5 \AA, 3.4 \AA$, and $3.4 \AA$, respectively, with no long-axis translation, as the angle between the molecules is changed from co-facial $\left(0^{\circ}\right)$ to $\mathrm{T}$-shaped $\left(90^{\circ}\right)$. Energy components are: total SAPT0 energy (black), SAPT0 electrostatics (blue), SAPT0 induction (green), SAPT0 dispersion (navy), and SAPT0 exchange (magenta). 

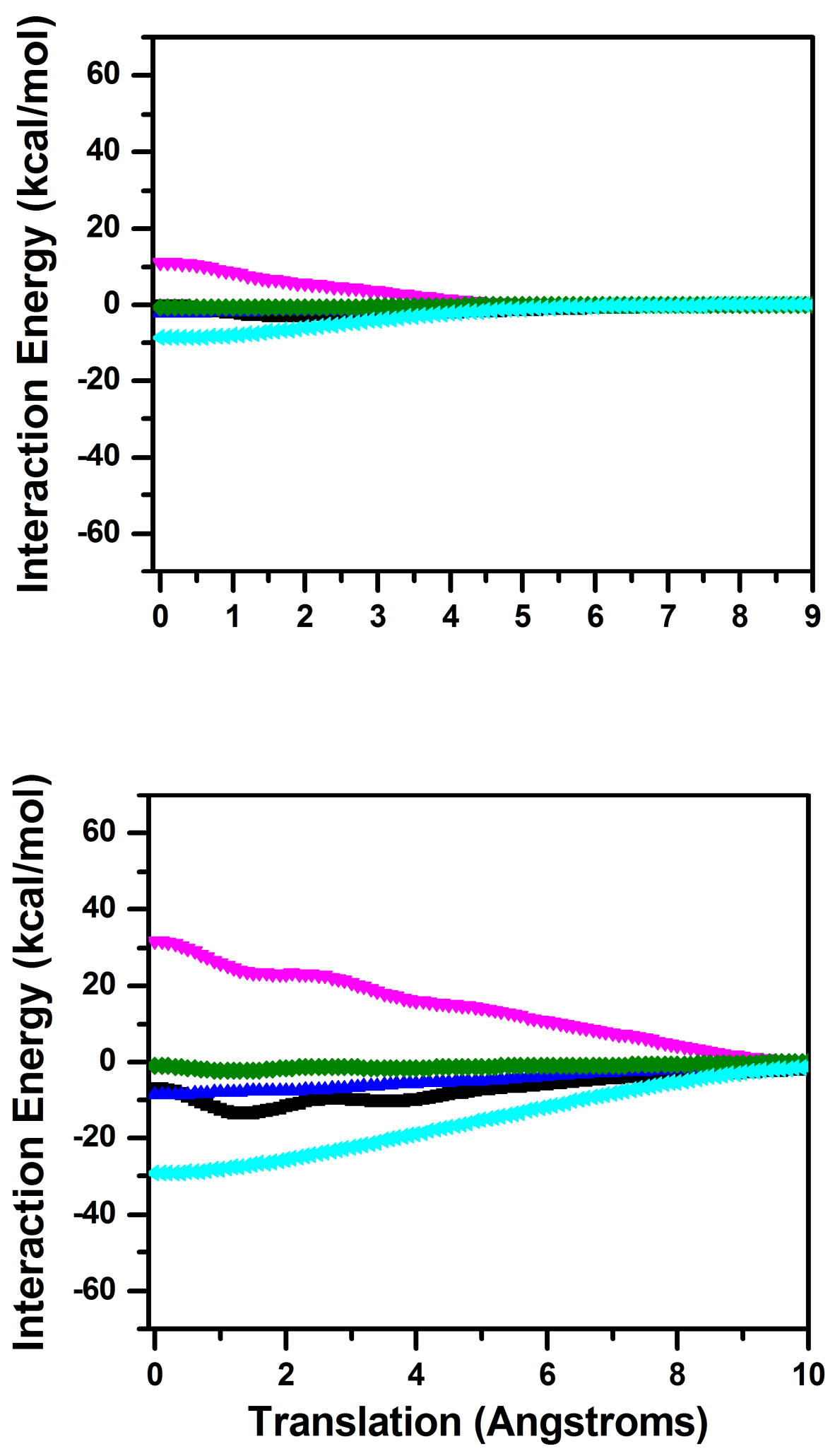

(Figure 5 continued on next page.) 


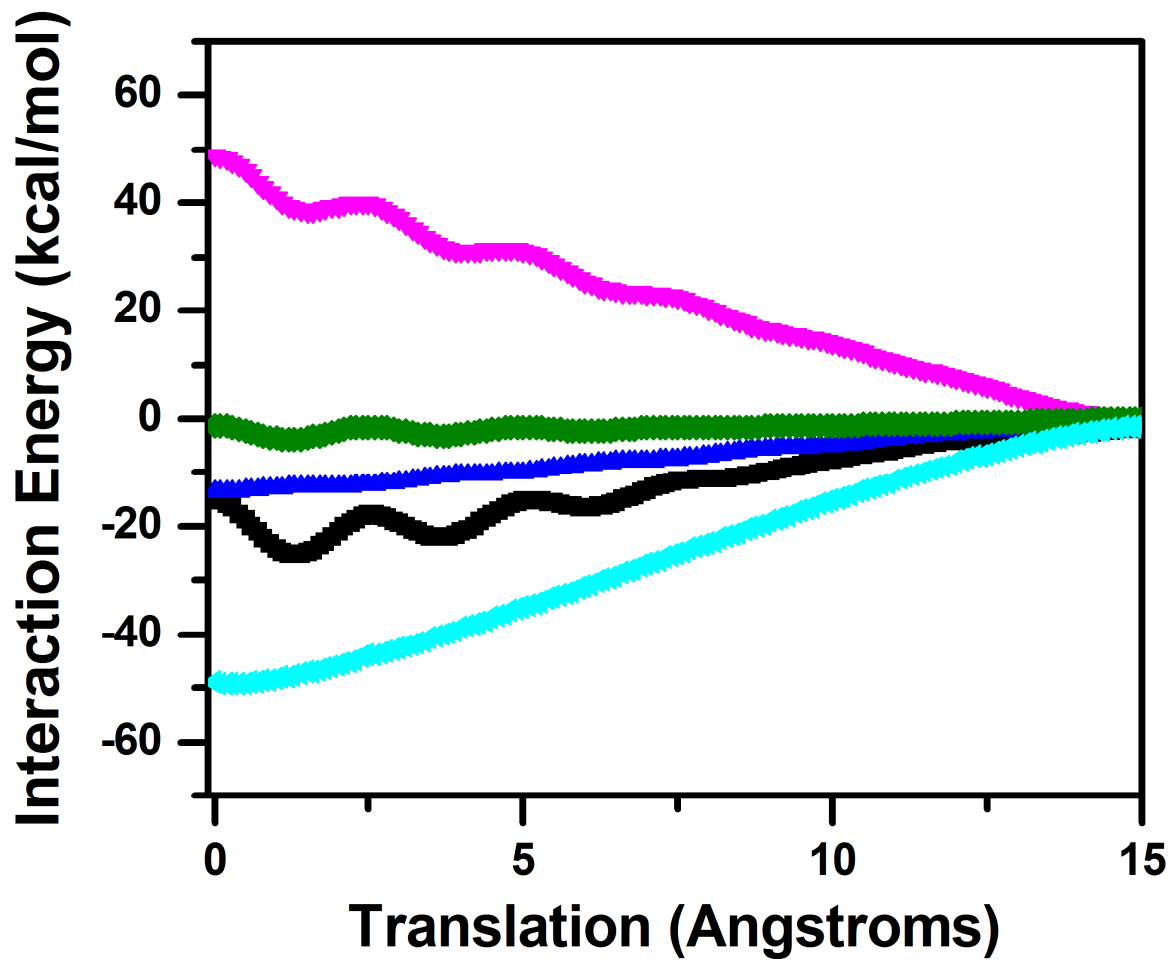

Figure 5. SAPT0 energy components for benzene (top), anthracene (center), and pentacene (bottom) at a separation distance of $3.5 \AA, 3.4 \AA$, and $3.4 \AA$, respectively, in a co-facial arrangement as the long-axis translation is varied from $0.0 \AA$ to no molecular overlap in $0.1 \AA$ increments. Energy components are: total SAPT0 energy (black), SAPT0 electrostatics (blue), SAPT0 induction (green), SAPT0 dispersion (cyan), and SAPT0 exchange (magenta).

Lastly, we consider co-facial dimers that undergo concurrent increased intermolecular separation and long-axis translation, see Figure 5. Contour plots provide additional details that are not otherwise readily apparent with traditional two-dimensional plots, which allows the extraction of information that might otherwise be missed. Here, we also shift our focus from benzene, anthracene, and pentacene to benzene, tetracene, and hexacene as interesting features become apparent in tetracene and hexacene that are more difficult to distinguish in anthracene and pentacene. From Figure 5, we find that the potential energy surfaces are indeed complex, with 
structures that are relatively stable at small intermolecular distances. For example, there is a stable benzene dimer $(-1.79 \mathrm{kcal} / \mathrm{mol})$ with only $2.8 \AA$ of interplane separation when one molecule is displaced by $5 \AA$; in this instance, the aromatic rings no longer possess spatial overlap but still strongly interact through their electron densities. In this configuration, the electrostatic component is strongly stabilizing $(-1.18 \mathrm{kcal} / \mathrm{mol})$, while the exchange $(1.81$ $\mathrm{kcal} / \mathrm{mol})$ and dispersion $(-2.22 \mathrm{kcal} / \mathrm{mol})$ terms nearly negate each other. Additionally, as the acene size increases to tetracene, there is the formation of a secondary well with the lowest point in the well corresponding to a separation distance of $3.3 \AA$. For tetracene, there also exists a third well forming at a long-axis displacement of $6.0 \AA$. This becomes more apparent in hexacene, where another stable configuration appears at about $8.0 \AA$ of long-axis translation. For each of these situations, the low-energy structures correspond to staggered co-facial arrangements reminiscent of the molecular packing found in TIPS-pentacene [6,13bis(triisopropylsilylethynyl)pentacene] - where the bridging carbon-carbon bonds of one molecule are interacting with the face of the fused rings on the adjacent molecule. ${ }^{3,73}$ While it is generally accepted that the sterics of the TIPS groups in TIPS-pentacene dominate the packing, preventing a herringbone packing motif, the non-bonded interactions act to fine tune this packing, resulting in the staggered, co-facial arrangement observed in the reported crystalline structure. 

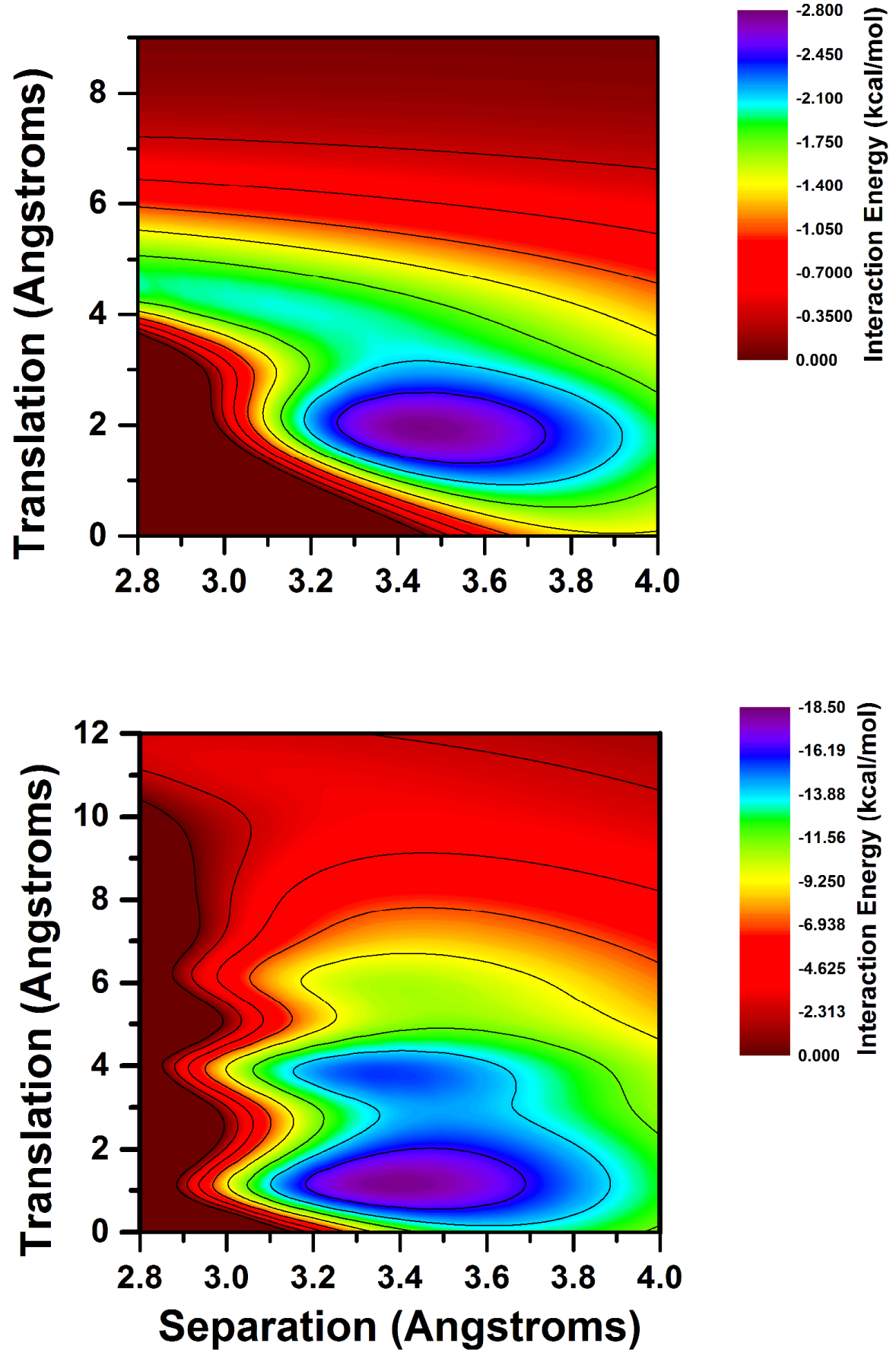

(Figure 5 continued on next page.) 

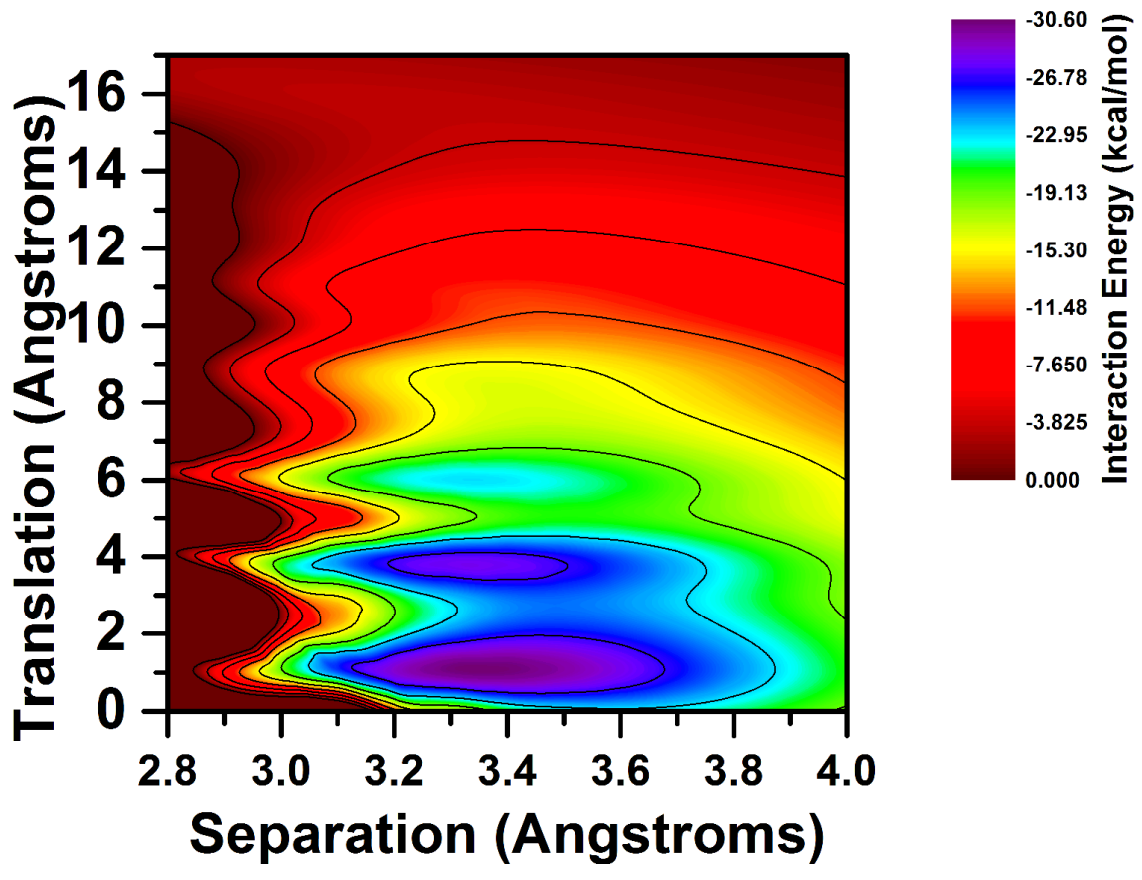

Figure 5. Contour plots of the total SAPT0 interaction energies for benzene (top), tetracene (center), and hexacene (bottom) for co-facial configurations as the separation distance and longaxis translation are varied. Note that the color scales have been adjusted for each plot so that qualitative differences are easier to distinguish. Dark red areas correspond to positive interaction energies.

\section{c) Charge Penetration Contribution to Non-Bonded Interactions}

In a traditional multipole electrostatics picture, implemented in most force fields using multipoles of rank 0 (i.e., charges) at atom centers, the electrostatic interactions in T-shaped dimers of the acenes are largely stabilizing, due to the positive-negative partial charge interactions that are trying to recreate the quadrupole-quadrupole interactions. In the co-facial dimers, the electrostatic interactions are destabilizing due to the negative-negative quadrupolequadrupole interactions, as shown in the last column of Table 2. However, CCSD(T) and SAPT calculations indicate that the co-facial dimer is in fact more stabilizing, ${ }^{23}$ which represents a 
qualitative discrepancy. From previous energy decomposition analysis studies ${ }^{34,46}$ and SAPT calculations, ${ }^{11}$ as well as has been shown here, the $E_{\text {elect }}$ component of the non-bonded interactions is more stabilizing in the co-facial configurations than in the corresponding T-shaped dimers, an effect due to charge penetration; this has been highlighted by Podeszwa and Szalewicz who state that for "stacked configurations, the stabilizing part of the electrostatic energy is exclusively due to the [penetration] effects." ${ }^{34}$

As the electron clouds of two monomers begin to overlap at small intermolecular distances, the multipole picture breaks down. This comes from the fact that in such instances there is a reduction of the screening of the nuclei in one monomer by its own electron distribution. Thus, there appears an attractive electron-nucleus interaction that increases rapidly as the overlap further increases; at the same time, $E_{\text {exch }}$ also increases rapidly and eventually overwhelms any stabilization due to charge penetration. This breakdown of the multipole approximation and the importance of charge penetration in benzene dimers are highlighted in Figure 6. At large separation distances, i.e., greater than $4 \AA$, the charge penetration contribution is negligible and the electrostatic contribution in the co-facial configuration is repulsive, which agrees very well with the multipole approximation. At separations less than $4 \AA$, the charge penetration is important, with the SAPT electrostatic contribution becoming strongly stabilizing in both dimer configurations, although more so for the co-facial case; this results in a qualitative difference between the multipole approximation and the SAPT electrostatics, as has been highlighted previously by Sherrill and co-workers. ${ }^{28}$ 


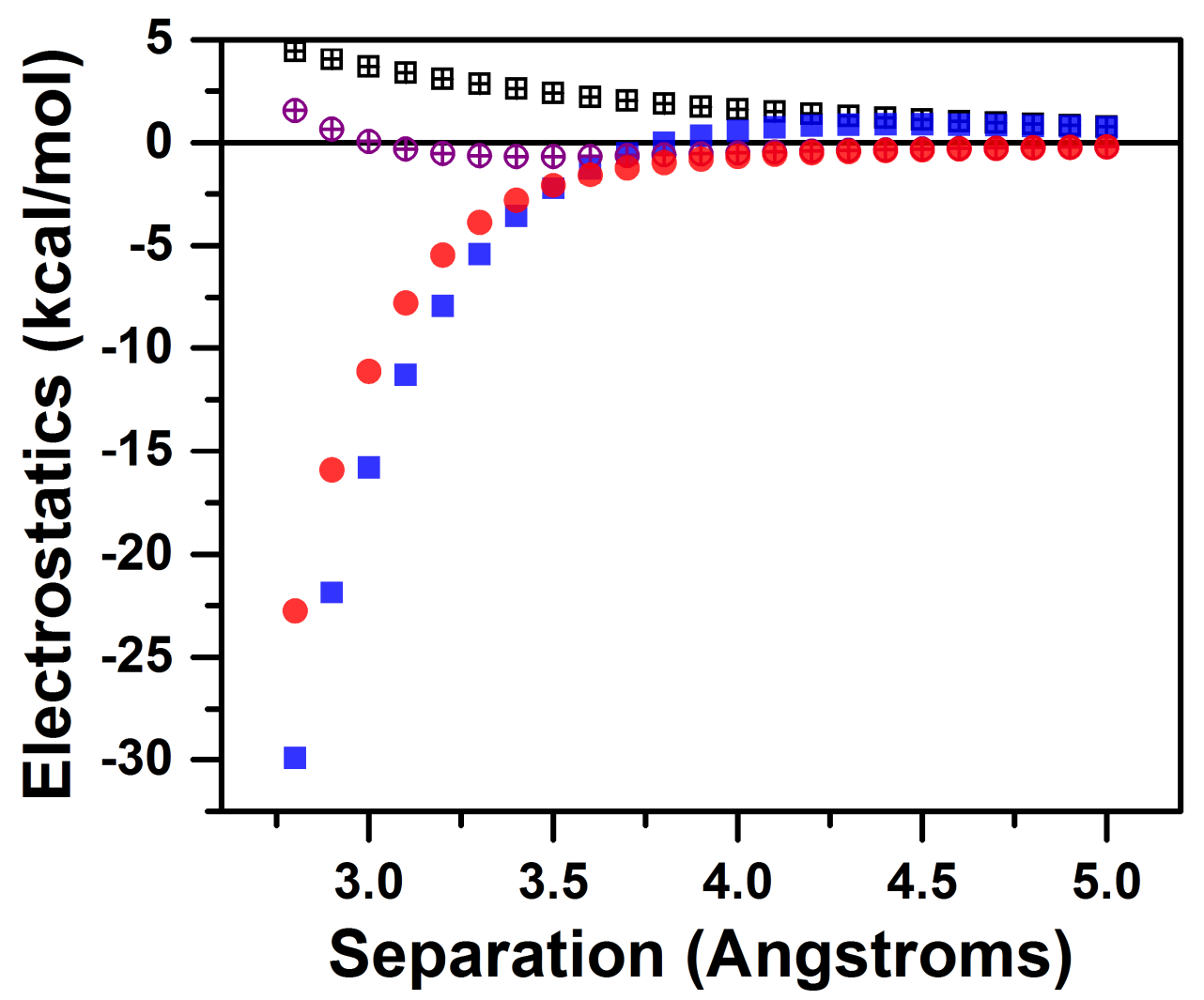

Figure 6. Electrostatic interaction energy in co-facial/eclipsed ( $\mathbf{\square}$; blue, black) and T-shaped (•; red, purple) benzene dimers with no long-axis translation, as calculated at the SAPT0 (solid) and DMA electrostatic (crossed) levels as a function of separation distance.

In order to quantify the charge penetration contribution in the oligoacene dimers, we evaluated the DMA multipole electrostatic interaction energy and the SAPT0 electrostatic interaction energy for each of the acene configurations considered above. We calculated the multipole interaction energy through the $5^{\text {th }}$ rank, e.g., charge-32-pole or octopole-quadrupole, to provide increased accuracy. Thus, the only significant difference between the SAPT0 electrostatic energy and the multipole electrostatic energy should be the contribution from charge penetration. In Figure 7, the difference between the multipole electrostatics and SAPT0 electrostatics is plotted 
for pentacene, with the resulting charge penetration contribution being more stabilizing than the SAPT0 electrostatics term itself; this result is due to the multipole interaction being destabilizing at smaller distances. For each of the lowest energy co-facial dimer configurations, the charge penetration contribution is calculated to be of the same size as the total SAPT0 interaction energy, which underlines the importance of this contribution (Table 3). It is interesting to note that for each system, the charge penetration turns on significantly (greater than $1 \mathrm{kcal} / \mathrm{mol}$ ) at a different point ( $4.0 \AA$ for co-facial benzene and $4.4 \AA$ for co-facial pentacene) while $E_{\text {exch }}$ begins to dominate at about $3.2 \AA$, resulting in a rapid decrease of the interaction energy. 

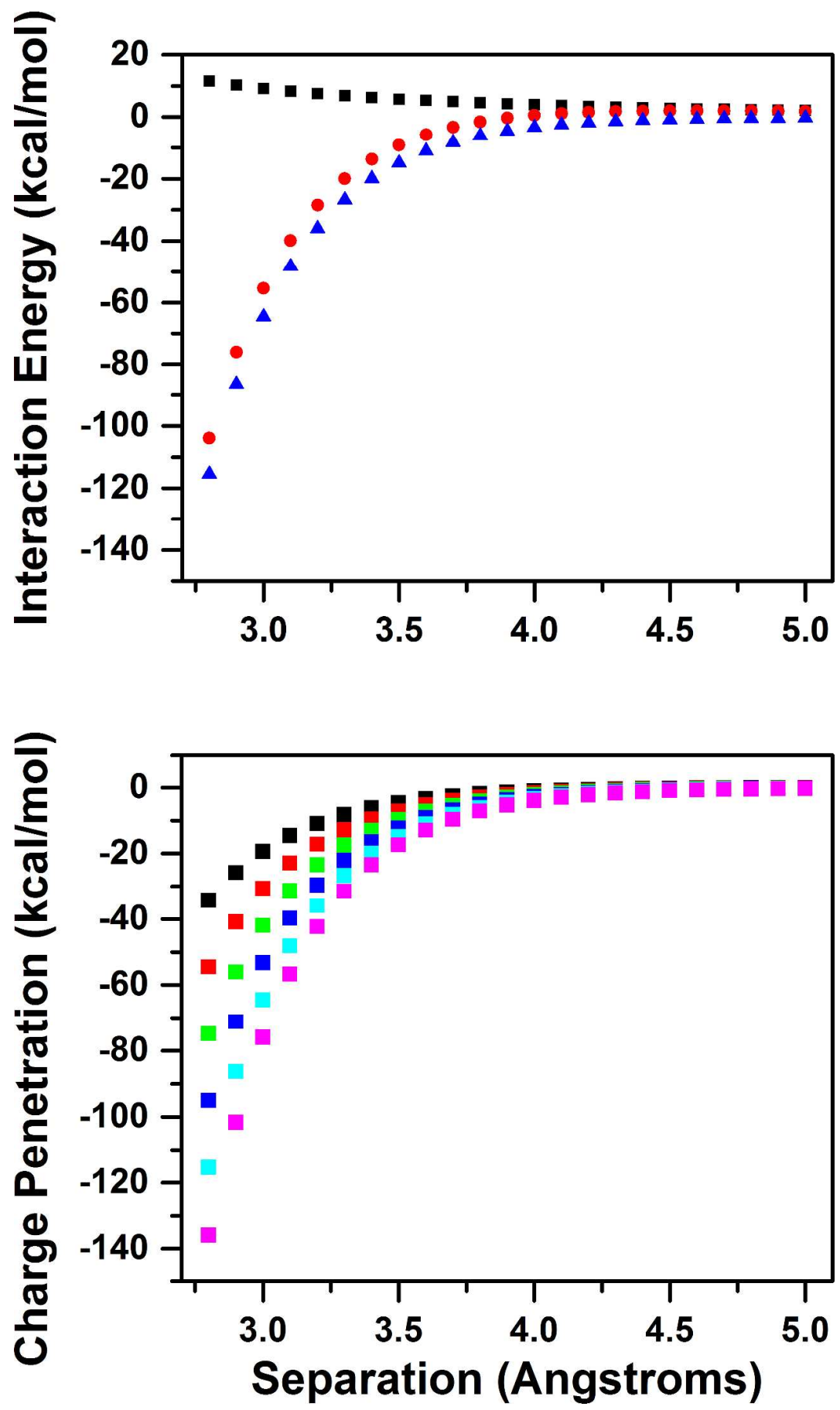

Figure 7. (Top) SAPT0 electrostatics interaction energy (red) and DMA electrostatic interactions energy (black) for pentacene in a co-facial configuration with no long-axis translation, as the separation distance changes in $0.1 \AA$ increments from $2.8 \AA$ to $5.0 \AA$. The charge penetration contribution (blue) is defined as the difference between the SAPT0 and DMA electrostatic energies. (Bottom) Charge penetration contributions for benzene (black), naphthalene (red), anthracene (green), tetracene (blue), pentacene (cyan), and hexacene (magenta) for eclipsed dimers as a function of separation distance. 
Table 3. SAPT0 interaction energies, DMA interaction energies, and charge penetration contribution in the most stable co-facial oligoacene dimers. All values in $\mathrm{kcal} / \mathrm{mol}$.

\begin{tabular}{l|ccc}
\hline (kcal/mol) & $E_{S A P T 0}$ & $E_{D M A}$ & $E_{c h p}$ \\
\hline Benzene & -2.82 & 1.34 & -6.15 \\
Naphthalene & -7.42 & 2.04 & -9.61 \\
Anthracene & -12.64 & 2.73 & -13.07 \\
Tetracene & -18.08 & 4.47 & -16.51 \\
Pentacene & -24.09 & 5.28 & -19.96 \\
Hexacene & -30.36 & 6.09 & -23.47 \\
\hline
\end{tabular}

We can further compare the charge penetration contribution for co-facial and T-shaped dimers in their respective lowest energy configurations, and the charge penetration contribution in the experimental crystalline dimers, see Figure 8 . For each set of systems, there is a linear increase in the charge penetration contribution as the acene length increases, with the amount of increase dependent on the dimer configurations. As one would expect because of the larger $\pi$ - $\pi$ overlap, the co-facial dimers present the largest charge penetration contribution; the T-shaped dimers also present a large charge penetration energy, due to the peripheral hydrogen atoms interacting with the face of the adjacent molecule. Interestingly, the charge penetration contribution in the experimental crystal dimers is much smaller than for either model configuration. This is due to the rotation in the plane of the backbone in the smaller acenes and a displacement along the short-axis of one of the molecules in the larger acenes, which reduces the overlap. Nevertheless, the charge penetration energy in the experimental structures still accounts for between $20 \%$ to $50 \%$ of the total interaction energy. Thus, charge penetration must be accounted for if prediction of the molecular packing of $\pi$-conjugated molecules is to be realized. 

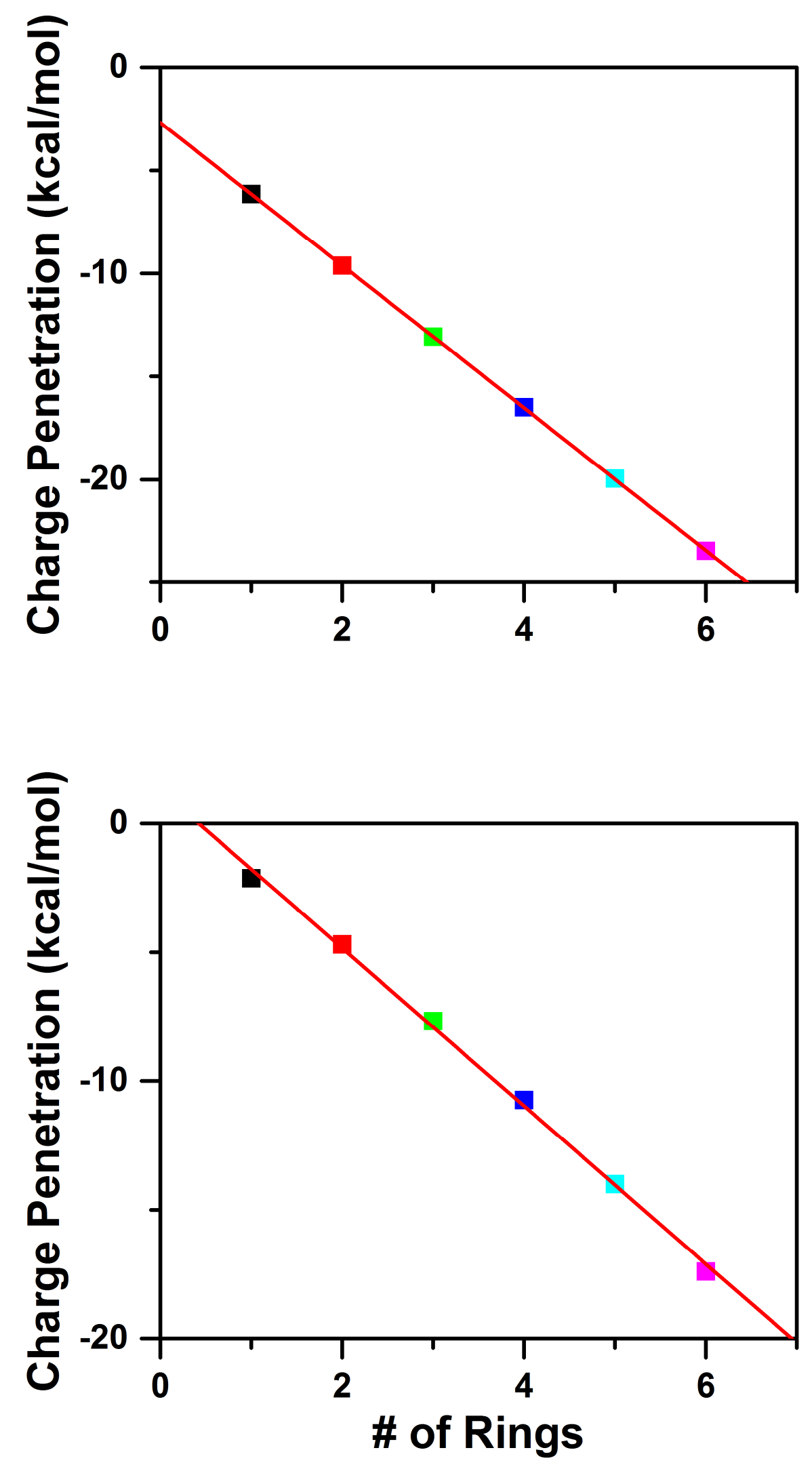

29

ACS Paragon Plus Environment 
(Figure 8 continued on next page.)

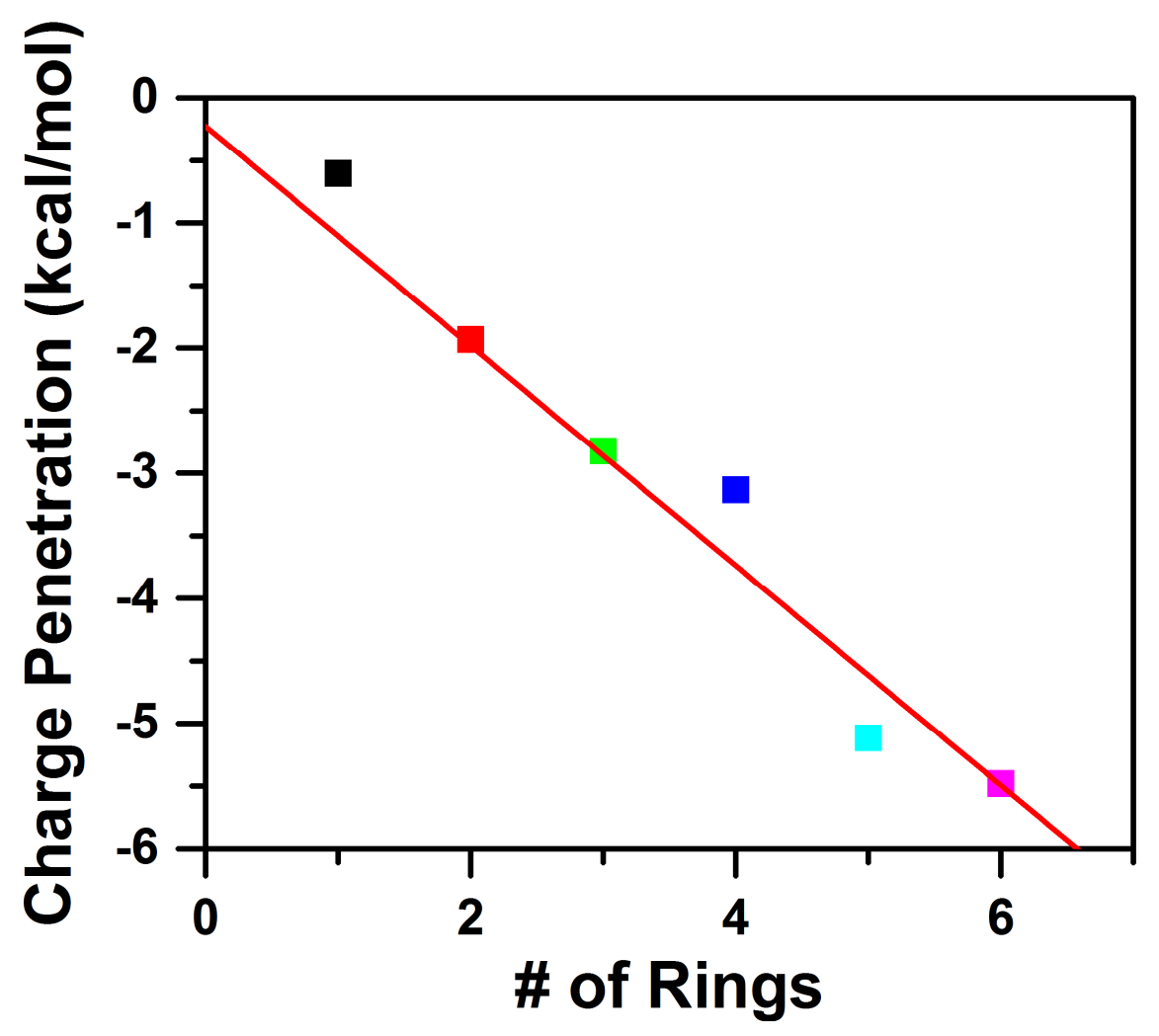

Figure 8. Evolution of the charge penetration contribution in the linear oligoacenes as a function of the number of fused rings for lowest energy co-facial dimers (top), lowest energy T-shaped dimers (center), and dimer configurations extracted from the experimental unit cells (bottom) of benzene (black), naphthalene (red), anthracene (green), tetracene (blue), pentacene (cyan), and hexacene (magenta).

\section{Synopsis}

We applied wave-function-based symmetry-adapted perturbation theory methodologies and distributed multipole analysis electrostatics approaches to the study of dimers of benzene through hexacene, to create potential energy surfaces under three transformations: (i) angle, (ii) separation, and (iii) long-axis translation. We find that the lowest-energy model dimers present a 
co-facial arrangement. In each instance, this configuration is significantly more stable than the configuration found in the experimental crystal structures; these results point out that additional interactions outside of those accessible from a dimer model are needed to accurately describe and eventually predict bulk packing. ${ }^{13,18,74}$ Considering the results obtained for the three transformations, the following conclusions can be drawn:

- Qualitatively identical behaviors for both intermonomer angle and separation transformations are observed as acene length is increased, with the primary difference being one of scale.

- Upon long-axis translation, there appear a series of peaks and valleys that correspond to the number of fused rings in each acene molecule. These valleys and peaks assist in explaining why the acenes pack in a staggered, herringbone motif instead of a perfectly aligned herringbone configuration.

- Upon combining transformations, smaller intermonomer separation distances become accessible as acene length increases, such as a $3.3 \AA$ separation in tetracene.

- For all of the acene systems, $E_{\text {elect }}, E_{\text {exch }}$, and $E_{\text {ind }}$ have a linear dependence on acene length while $E_{\text {disp }}$ and thus $E_{S A P T 0}$ have a non-linear dependence.

- As the dimer configurations change, there is a smooth evolution of the non-covalent interactions; however, it is evident that a two-body approach is not sufficient to capture the intricate interactions in non-polar organic crystals. 
We also specifically explored the charge penetration stabilization that occurs as electron densities overlap at short distances. The multipole approximation is seen to breakdown at separation distances shorter than $4 \AA$, with this evolution not restricted to co-facial systems but also occurring for the T-shaped dimers. With respect to the intermolecular total interaction energy, for both the model systems and the dimers extracted from the experimental crystal structures, the charge penetration is calculated to be a stabilizing force of similar size.

Our results confirm that an accurate prediction of the interactions among $\pi$-conjugated molecules requires proper account of charge penetration and three-body interactions. ${ }^{75}$ Overall, the potential energy surfaces for the oligoacene dimers expose the challenges facing molecular crystal engineering, with the small energy differences among rather different configurations of $\pi$ conjugated dimers illustrating why polymorphs are such common features of these systems. The results also provide optimism in the full breadth of synthetic chemistry to coax molecules into configurations optimal for organic semiconductor applications through judicious choice of chemical functionality. 
Acknowledgements. This work has been supported by King Abdullah University of Science and Technology (KAUST), the KAUST Competitive Research Grant program, and the Office of Naval Research Global (Award N62909-15-1-2003). We acknowledge the IT Research Computing Team and Supercomputing Laboratory at KAUST for providing computational and storage resources. This work has also used the computing resources of the Garnet, Spirit, and Copper supercomputing systems through the DoD HPCMP. We wish to thank Prof. C. David Sherrill, Dr. Rob Parrish, and Mr. Trent Parker for technical assistance and stimulating discussions.

Supporting Information Available: A comparison of different multipole expansions, additional plots of the total SAPT0 interaction energy and interaction components as a function of angle, separation, and long-axis translation and additional plots of the charge penetration contribution for benzene, naphthalene, anthracene, tetracene, pentacene, and hexacene are available in the Supporting Information. This material is available free of charge via the Internet at http://pubs.acs.org. 


\section{References}

1. Pope, M.; Swenberg, C. E., Electronic Processes in Organic Crystals and Polymers. 1999.

2. McGarry, K. A.; Xie, W.; Sutton, C.; Risko, C.; Wu, Y.; Young, V. G.; Brédas, J.-L.; Frisbie, C. D.; Douglas, C. J., Rubrene-Based Single-Crystal Organic Semiconductors: Synthesis, Electronic Structure, and Charge-Transport Properties. Chem. Mater. 2013, 25 (11), 2254-2263.

3. Ryno, S. M.; Risko, C.; Brédas, J.-L., Impact of Molecular Packing on Electronic Polarization in Organic Crystals: The Case of Pentacene vs TIPS-Pentacene. J. Am. Chem. Soc. 2014, 136 (17), 6421-6427.

4. Wang, B.; Truhlar, D. G., Including Charge Penetration Effects in Molecular Modeling. J. Chem. Theory Comput. 2010, 6 (11), 3330-3342.

5. Freitag, M. A.; Gordon, M. S.; Jensen, J. H.; Stevens, W. J., Evaluation of Charge Penetration Between Distributed Multipolar Expansions. J. Chem. Phys. 2000, 112 (17), 7300-7306.

6. Arnstein, S. A.; Sherrill, C. D., Substituent Effects in Parallel-Displaced $\pi-\pi$ Interactions. Phys. Chem. Chem. Phys. 2008, 10 (19), 2646-2655.

7. Wheeler, S. E.; Bloom, J. W. G., Toward a More Complete Understanding of Noncovalent Interactions Involving Aromatic Rings. J. Phys. Chem. A 2014, 118 (32), 6133-6147.

8. Wheeler, S. E.; Bloom, J. W. G., Anion- $\pi$ Interactions and Positive Electrostatic Potentials of $\mathrm{N}$-Heterocycles Arise from the Positions of the Nuclei, not Changes in the $\pi$-Electron Distribution. Chem. Commun. (Cambridge, U. K.) 2014, 50 (76), 11118-11121.

9. Sherrill, C. D.; Sumpter, B. G.; Sinnokrot, M. O.; Marshall, M. S.; Hohenstein, E. G.; Walker, R. C.; Gould, I. R., Assessment of Standard Force Field Models Against High-Quality Ab Initio Potential Curves for Prototypes of $\pi-\pi, \mathrm{CH} / \pi$, and $\mathrm{SH} / \pi$ Interactions. J. Comput. Chem. 2009, 30 (14), 2187-2193.

10. Geng, Y.; Takatani, T.; Hohenstein, E. G.; Sherrill, C. D., Accurately Characterizing the $\pi-\pi$ Interaction Energies of Indole-Benzene Complexes. J. Phys. Chem. A 2010, 114 (10), 35763582.

11. Hohenstein, E. G.; Sherrill, C. D., Density fitting and Cholesky decomposition approximations in symmetry-adapted perturbation theory: Implementation and application to probe the nature of $\pi-\pi$ interactions in linear acenes. J. Chem. Phys. 2010, 132 (18), 184111.

12. Ringer, A. L.; Sherrill, C. D., First Principles Computation of Lattice Energies of Organic Solids: The Benzene Crystal. Chem. Eur. J. 2008, 14 (8), 2542-2547.

13. Szalewicz, K., Determination of Structure and Properties of Molecular Crystals from First Principles. Acc. Chem. Res. 2014, 47 (11), 3266-3274.

14. Sherrill, C. D., Energy Component Analysis of $\pi$ Interactions. Acc. Chem. Res. 2013, 46 (4), 1020-1028.

15. Bloom, J. W. G.; Wheeler, S. E., Taking the Aromaticity out of Aromatic Interactions. Angew. Chem., Int. Ed. 2011, 50 (34), 7847-7849.

16. Note that most force fields include a Lennard-Jones term that does account for the charge penetration in an effective manner.

17. Piquemal, J.-P.; Cisneros, G. A.; Reinhardt, P.; Gresh, N.; Darden, T. A., Towards a force field based on density fitting. J. Chem. Phys. 2006, 124 (10), 104101-104101. 
18. Wang, Q.; Rackers, J. A.; He, C.; Qi, R.; Narth, C.; Lagardere, L.; Gresh, N.; Ponder, J. W.; Piquemal, J.-P.; Ren, P., General Model for Treating Short-Range Electrostatic Penetration in a Molecular Mechanics Force Field. J. Chem. Theory Comput. 2015, 11 (6), 2609-2618.

19. Cisneros, G. A.; Tholander, S. N.-I.; Parisel, O.; Darden, T. A.; Elking, D.; Perera, L.; Piquemal, J. P., Simple Formulas for Improved Point-Charge Electrostatics in Classical Force Fields and Hybrid Quantum Mechanical/Molecular Mechanical Embedding. Int. J. Quantum Chem. 2008, 108 (11), 1905-1912.

20. McDaniel, J. G.; Schmidt, J. R., Physically-Motivated Force Fields from Symmetry-Adapted Perturbation Theory. J. Phys. Chem. A 2013, 117 (10), 2053-2066.

21. Choi, E.; McDaniel, J. G.; Schmidt, J. R.; Yethiraj, A., First-Principles, Physically Motivated Force Field for the Ionic Liquid [BMIM][BF4]. J. Phys. Chem. Lett. 2014, 5 (15), 2670-2674.

22. Sutton, C.; Risko, C.; Bredas, J.-L., Non-Covalent Intermolecular Interactions in Organic Electronic Materials: Implications for the Molecular Packing vs. Electronic Properties of Acenes. Chem. Mater. 2015, 28 (1), 3-16.

23. Sinnokrot, M. O; Sherrill, C. D., Highly Accurate Coupled Cluster Potential Energy Curves for the Benzene Dimer: Sandwich, T-Shaped, and Parallel-Displaced Configurations. J. Phys. Chem. A 2004, 108 (46), 10200-10207.

24. Sinnokrot, M. O.; Sherrill, C. D., Substituent Effects in $\pi-\pi$ Interactions: Sandwich and TShaped Configurations. J. Am. Chem. Soc. 2004, 126 (24), 7690-7697.

25. Sinnokrot, M. O.; Sherrill, C. D., High-Accuracy Quantum Mechanical Studies of $\pi-\pi$ Interactions in Benzene Dimers. J. Phys. Chem. A 2006, 110 (37), 10656-10668.

26. Ringer, A. L.; Figgs, M. S.; Sinnokrot, M. O.; Sherrill, C. D., Aliphatic C $-\mathrm{H} / \pi$ Interactions: Methane-Benzene, Methane-Phenol, and Methane-Indole Complexes. J. Phys. Chem. A 2006, 110 (37), 10822-10828.

27. Marshall, M. S.; Steele, R. P.; Thanthiriwatte, K. S.; Sherrill, C. D., Potential Energy Curves for Cation $-\pi$ Interactions: Off-Axis Configurations Are Also Attractive. J. Phys. Chem. A 2009, 113 (48), 13628-13632.

28. Hohenstein, E. G.; Duan, J.; Sherrill, C. D., Origin of the Surprising Enhacement of Electrostatic Energies by Electron-Donating Substituents in Substituted Sandwich Benzene Dimers. J. Am. Chem. Soc. 2011, 133, 13244-13247.

29. Wheeler, S. E.; Houk, K. N., Substituent Effects in the Benzene Dimer are Due to Direct Interactions of the Substituents with the Unsubstituted Benzene. J. Am. Chem. Soc. 2008, 130 (33), 10854-10855.

30. Wheeler, S. E.; McNeil, A. J.; Müller, P.; Swager, T. M.; Houk, K. N., Probing Substituent Effects in Aryl-Aryl Interactions Using Stereoselective Diels-Alder Cycloadditions. J. Am. Chem. Soc. 2010, 132 (10), 3304-3311.

31. Wheeler, S. E., Understanding Substituent Effects in Noncovalent Interactions Involving Aromatic Rings. Acc. Chem. Res. 2012, 46 (4), 1029-1038.

32. Jeziorski, B.; Moszynski, R.; Szalewicz, K., Perturbation Theory Approach to Intermolecular Potential Energy Surfaces of van der Waals Complexes. Chem. Rev. 1994, 94 (7), 1887-1930.

33. Podeszwa, R.; Bukowski, R.; Szalewicz, K., Potential Energy Surface for the Benzene Dimer and Perturbational Analysis of $\pi-\pi$ Interactions. J. Phys. Chem. A 2006, 110 (34), $10345-$ 10354. 
34. Podeszwa, R.; Szalewicz, K., Physical origins of interactions in dimers of polycyclic aromatic hydrocarbons. Phys. Chem. Chem. Phys. 2008, 10 (19), 2735-2746.

35. The separation distance is defined using a weighted function where, in the co-facial arrangement, the distance is defined as face-to-face and in the T-shaped arrangement the distance is defined as the face-to-hydrogen distance.

36. Civalleri, B.; Zicovich-Wilson, C. M.; Valenzano, L.; Ugliengo, P., B3LYP Augmented with an Empirical Dispersion Term (B3LYP-D*) as Applied to Molecular Crystals. Cryst. Eng. Comm. 2008, 10 (4), 405-410.

37. Grimme, S., Semiempirical GGA-Type Density Functional Constructed with a Long-Range Dispersion Correction. J. Comput. Chem. 2006, 27 (15), 1787-1799.

38. Grimme, S., Accurate Description of van der Waals Complexes by Density Functional Theory Including Empirical Corrections. J. Comput. Chem. 2004, 25 (12), 1463-1473.

39. Grimme, S.; Antony, J.; Schwabe, T.; Muck-Lichtenfeld, C., Density Functional Theory with Dispersion Corrections for Supramolecular Structures, Aggregates, and Complexes of (Bio)organic Molecules. Org. Biomol. Chem. 2007, 5 (5), 741-758.

40. Jurečka, P.; Černý, J.; Hobza, P.; Salahub, D. R., Density Functional Theory Augmented with an Empirical Dispersion Term. Interaction Energies and Geometries of 80 Noncovalent Complexes Compared with Ab Initio Quantum Mechanics Calculations. J. Comput. Chem. 2007, $28(2), 555-569$.

41. Wu, Q.; Yang, W., Empirical Correction to Density Functional Theory for van der Waals Interactions. J. Chem. Phys. 2002, 116 (2), 515-524.

42. Zimmerli, U.; Parrinello, M.; Koumoutsakos, P., Dispersion Corrections to Density Functionals for Water Aromatic Interactions. J. Chem. Phys. 2004, 120 (6), 2693-2699.

43. Chai, J. D.; Head-Gordon, M., Long-Range Corrected Hybrid Density Functionals with Damped Atom-Atom Dispersion Corrections. Phys. Chem. Chem. Phys. 2008, 10, 6615.

44. Raghavachari, K.; Trucks, G. W.; Pople, J. A.; Head-Gordon, M., A Fifth-Order Perturbation Comparison of Electron Correlation Theories. Chem. Phys. Lett. 1989, 157 (6), 479483.

45. Grimme, S.; Antony, J.; Ehrlich, S.; Krieg, H., A Consistent and Accurate Ab Initio Parametrization of Density Functional Dispersion Correction (DFT-D) for the 94 Elements H-Pu. J. Chem. Phys. 2010, 132 (15), 154104.

46. Grimme, S., Improved Second-Order Møller-Plesset Perturbation Theory by Separate Scaling of Parallel- and Antiparallel-Spin Pair Correlation Energies. J. Chem. Phys. 2003, 118 (20), 9095-9102.

47. Takatani, T.; Hohenstein, E. G.; Sherrill, C. D., Improvement of the Coupled-Cluster Singles and Doubles Method via Scaling Same- and Opposite-Spin Components of the Double Excitation Correlation Energy. J. Chem. Phys. 2008, 128 (12), 124111.

48. Hill, J. G.; Platts, J. A.; Werner, H.-J., Calculation of Intermolecular Interactions in the Benzene Dimer using Coupled-Cluster and Local Electron Correlation Methods. Phys. Chem. Chem. Phys. 2006, 8 (35), 4072-4078.

49. Hesselmann, A.; Jansen, G.; Schutz, M., Density-Functional Theory-Symmetry-Adapted Intermolecular Perturbation Theory with Density Fitting: A New Efficient Method to Study Intermolecular Interaction Energies. J. Chem. Phys. 2005, 122, 014103.

50. Morokuma, K., Molecular Orbital Studies of Hydrogen Bonds. III. $\mathrm{C}=\mathrm{O} \cdots \mathrm{H}-\mathrm{O}$ Hydrogen Bond in $\mathrm{H} 2 \mathrm{CO} \cdots \mathrm{H} 2 \mathrm{O}$ and $\mathrm{H} 2 \mathrm{CO} \cdots 2 \mathrm{H} 2 \mathrm{O}$. J. Chem. Phys. 1971, 55 (3), 1236-1244. 
51. Kitaura, K.; Morokuma, K., A New Energy Decomposition Scheme for Molecular Interactions within the Hartree-Fock Approximation. Int. J. Quantum Chem. 1976, 10 (2), 325340.

52. Grimme, S., Do Special Noncovalent $\pi-\pi$ Stacking Interactions Really Exist? Angew. Chem., Int. Ed. 2008, 47 (18), 3430-3434.

53. Turney, J. M.; Simmonett, A. C.; Parrish, R. M.; Hohenstein, E. G.; Evangelista, F. A.; Fermann, J. T.; Mintz, B. J.; Burns, L. A.; Wilke, J. J.; Abrams, M. L.; Russ, N. J.; Leininger, M. L.; Janssen, C. L.; Seidl, E. T.; Allen, W. D.; Schaefer, H. F.; King, R. A.; Valeev, E. F.; Sherrill, C. D.; Crawford, T. D., Psi4: An Open-Source Ab Initio Electronic Structure Program. WIREs Comput. Mol. Sci. 2012, 2 (4), 556-565.

54. Parker, T. M.; Burns, L. A.; Parrish, R. M.; Ryno, A. G.; Sherrill, C. D., Levels of Symmetry Adapted Perturbation Theory (SAPT). I. Efficiency and Performance for Interaction Energies. $J$. Chem. Phys. 2014, 140 (9), 094106.

55. van der Avoird, A.; Podeszwa, R.; Szalewicz, K.; Leforestier, C.; van Harrevelt, R.; Bunker, P. R.; Schnell, M.; von Helden, G.; Meijer, G., Vibration-rotation-tunneling states of the benzene dimer: an ab initio study. Phys. Chem. Chem. Phys. 2010, 12 (29), 8219-8240.

56. Werner, H.-J.; Knowles, P. J.; Knizia, G.; Manby, F. R.; Schütz, M.; Celani, P.; Korona, T.; Lindh, R.; Mitrushenkov, A.; Rauhut, G.; Shamasundar, K. R.; Adler, T. B.; Amos, R. D.; Bernhardsson, A.; Berning, A.; Cooper, D. L.; Deegan, M. J. O.; Dobbyn, A. J.; Eckert, F.; Goll, E.; Hampel, C.; Hesselmann, A.; Hetzer, G.; Hrenar, T.; Jansen, G.; Köppl, C.; Liu, Y.; Lloyd, A. W.; Mata, R. A.; May, A. J.; McNicholas, S. J.; Meyer, W.; Mura, M. E.; Nicklass, A.; O'Neill, D. P.; Palmieri, P.; Peng, D.; Pflüger, K.; Pitzer, R.; Reiher, M.; Shiozaki, T.; Stoll, H.; Stone, A. J.; Tarroni, R.; Thorsteinsson, T.; Wang, M. MOLPRO, Version 2012.1, a Package of Ab Initio Programs, Cardiff, UK, 2012.

57. Stone, A. J., The Theory of Intermolecular Forces. Clarendon Press: Oxford, 1996.

58. Katrusiak, A.; Podsiadło, M.; Budzianowski, A., Association $\mathrm{CH}^{\cdots} \pi$ and No van der Waals Contacts at the Lowest Limits of Crystalline Benzene I and II Stability Regions. Cryst. Growth Des. 2010, 10 (8), 3461-3465.

59. Brock, C. P.; Dunitz, J. D., Temperature Dependence of Thermal Motion in Crystalline Naphthalene. Acta Crystallogr., Sect. B: Struct. Sci. 1982, 38, 2218.

60. Brock, C. P.; Dunitz, J. D., Temperature Dependence of Thermal Motion in Crystalline Anthracene. Acta Crystallogr., Sect. B: Struct. Sci. 1990, 46, 795.

61. Holmes, D.; Kumaraswamy, S.; Matzger, A. J.; Vollhardt, K. P. C., On the Nature of Nonplanarity in the [N]Phenylenes. Chem. Eur. J. 1999, 5, 3399-3412.

62. Mattheus, C. C.; Dros, A. B.; Baas, J.; Meetsma, A.; Boer, J. L. d.; Palstra, T. T. M., Polymorphism in Pentacene. Acta Crystallogr., Sect. C: Cryst. Struct. Commun. 2001, 57, 939.

63. Watanabe, M.; Chang, Y. J.; Liu, S.-W.; Chao, T.-H.; Goto, K.; Islam, M. M.; Yuan, C.-H.; Tao, Y.-T.; Shinmyozu, T.; Chow, T. J., The Synthesis, Crystal Structure and Charge-Transport Properties of Hexacene. Nature Chem. 2012, 4 (7), 574-578.

64. Frisch, M. J.; Trucks, G. W.; Schlegel, H. B.; Scuseria, G. E.; Robb, M. A.; Cheeseman, J. R.; Scalmani, G.; Barone, V.; Mennucci, B.; Petersson, G. A.; Nakatsuji, H.; Caricato, M.; Li, X.; Hratchian, H. P.; Izmaylov, A. F.; Bloino, J.; Zheng, G.; Sonnenberg, J. L.; Hada, M.; Ehara, M.; Toyota, K.; Fukuda, R.; Hasegawa, J.; Ishida, M.; Nakajima, T.; Honda, Y.; Kitao, O.; Nakai, H.; Vreven, T.; Montgomery Jr., J. A.; Peralta, J. E.; Ogliaro, F.; Bearpark, M. J.; Heyd, J.; Brothers, E. N.; Kudin, K. N.; Staroverov, V. N.; Kobayashi, R.; Normand, J.; Raghavachari, K.; Rendell, A. P.; Burant, J. C.; Iyengar, S. S.; Tomasi, J.; Cossi, M.; Rega, N.; Millam, N. J.; 
Klene, M.; Knox, J. E.; Cross, J. B.; Bakken, V.; Adamo, C.; Jaramillo, J.; Gomperts, R.; Stratmann, R. E.; Yazyev, O.; Austin, A. J.; Cammi, R.; Pomelli, C.; Ochterski, J. W.; Martin, R. L.; Morokuma, K.; Zakrzewski, V. G.; Voth, G. A.; Salvador, P.; Dannenberg, J. J.; Dapprich, S.; Daniels, A. D.; Farkas, Ö.; Foresman, J. B.; Ortiz, J. V.; Cioslowski, J.; Fox, D. J. Gaussian 09, Gaussian, Inc.: Wallingford, CT, USA, 2009.

65. Here, the distance between the monomers as a function of angle is defined using a weighted linear function, where in co-facial arrangements the distance is defined as the separation between molecular planes (COM-to-COM), and in the T-shaped configuration as the distance between the peripheral hydrogen of one monomer and the plane of the other (edge-to-COM). The function is weighted such that in the co-facial configuration the COM-to-COM distance has a weight of 1 and the edge-to-COM distance has a weight of 0 , while in the T-shaped configuration the COMto-COM distance has a weight of 0 and the edge-to-COM distance has a weight of 1 . This maintains a constant separation distance in the manner of a sliding pendulum.

66. Jorgensen, W. L.; Severance, D. L., Aromatic-aromatic interactions: free energy profiles for the benzene dimer in water, chloroform, and liquid benzene. J. Am. Chem. Soc. 1990, 112 (12), 4768-4774.

67. The rate at which the interaction energy decreases will be dependent on the separation distance. At separation distances up to 4 angstroms, there will be exponential decrease as charge penetration will be a large contribution. At larger separations, there will be $1 / R^{\wedge} n$ dependence due to dispersion and various electrostatic multipole interactions.

68. The distance dependence of the electrostatics and induction terms are dependent on the rank of the interacting multipoles with electrostatics also having an exponential dependence on the overlap for the charge penetration. The distance dependence takes the form $1 / R^{\wedge}(1+L+M)$ where $\mathrm{L}$ and $\mathrm{M}$ are the rank of the interacting multipoles, i.e., charge is rank 0 , dipole is rank 1 , charge induced dipole is rank 2 , etc.

69. Kennedy, M. R.; McDonald, A. R.; DePrince, A. E.; Marshall, M. S.; Podeszwa, R.; Sherrill, C. D., Communication: Resolving the Three-Body Contribution to the Lattice Energy of Crystalline Benzene: Benchmark Results from Coupled-Cluster Theory. J. Chem. Phys. 2014, 140 (12), 121104.

70. Anatole von Lilienfeld, O.; Tkatchenko, A., Two- and Three-Body Interatomic Dispersion Energy Contributions to Binding in Molecules and Solids. J. Chem. Phys. 2010, 132 (23), 234109.

71. Wen, S.; Beran, G. J. O., Accurate Molecular Crystal Lattice Energies from a Fragment QM/MM Approach with On-the-Fly Ab Initio Force Field Parametrization. J. Chem. Theory Comput. 2011, 7 (11), 3733-3742.

72. Podeszwa, R.; Rice, B. M.; Szalewicz, K., Predicting Structure of Molecular Crystals from First Principles. Phys. Rev. Lett. 2008, 101 (11), 115503.

73. Griffith, O. L.; Anthony, J. E.; Jones, A. G.; Lichtenberger, D. L., Electronic Properties of Pentacene versus Triisopropylsilylethynyl-Substituted Pentacene: Environment-Dependent Effects of the Silyl Substituent. J. Am. Chem. Soc. 2010, 132 (2), 580-586.

74. Tafipolsky, M.; Engels, B., Accurate Intermolecular Potentials with Physically Grounded Electrostatics. J. Chem. Theory Comput. 2011, 7 (6), 1791-1803.

75. While three-body interactions are necessary for the prediction of nonpolar material packing, this is not necessarily the case for polar materials where electrostatic effects can dominate the packing. However, inclusion of such terms could be potentially useful is discriminating between packings with similar energies. 
28

30

31

32

33

34

35

36

37

38

39

40

41

43

44

45

46

47

48

49

51

52

53

54

55

57

58

59 


\section{Table of Contents Figure}

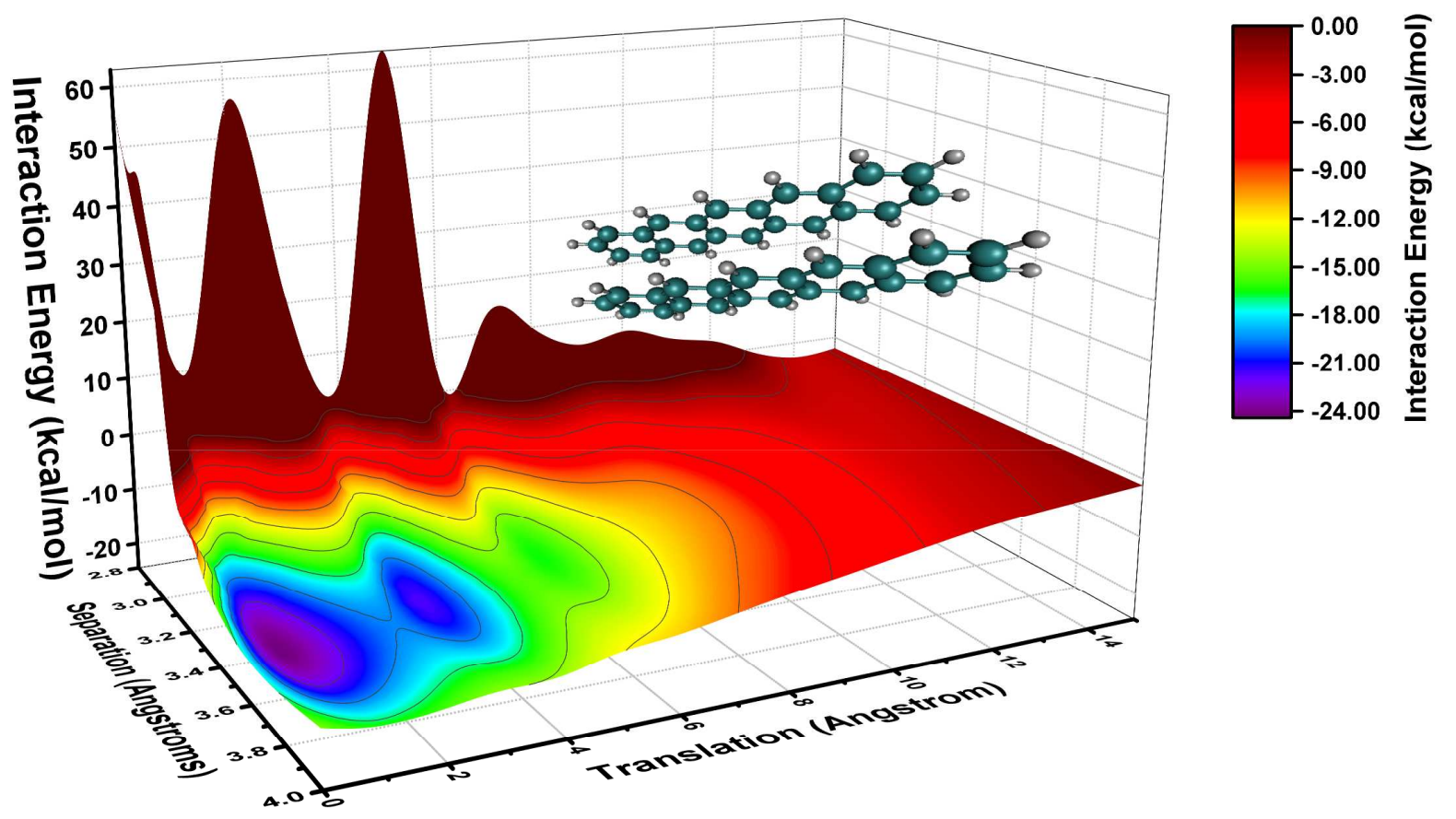

